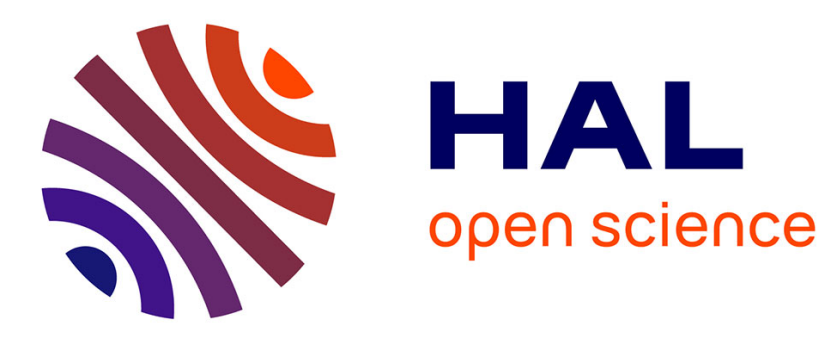

\title{
Damage Measurements via DIC
}

François Hild, Amine Bouterf, Stéphane Roux

\section{To cite this version:}

François Hild, Amine Bouterf, Stéphane Roux. Damage Measurements via DIC: From Physical to Mechanical Damage. International Journal of Fracture, 2015, IJF at 50, 191 (1-2), pp.77-105. 10.1007/s10704-015-0004-7 . hal-01166736

\section{HAL Id: hal-01166736 https://hal.science/hal-01166736}

Submitted on 23 Jun 2015

HAL is a multi-disciplinary open access archive for the deposit and dissemination of scientific research documents, whether they are published or not. The documents may come from teaching and research institutions in France or abroad, or from public or private research centers.
L'archive ouverte pluridisciplinaire HAL, est destinée au dépôt et à la diffusion de documents scientifiques de niveau recherche, publiés ou non, émanant des établissements d'enseignement et de recherche français ou étrangers, des laboratoires publics ou privés. 


\title{
Damage Measurements via DIC \\ From Physical to Mechanical Damage
}

\author{
François Hild • Amine Bouterf • Stéphane \\ Roux
}

Received: January 15, 2015/ Accepted: tomorrow

\begin{abstract}
The present paper is devoted to the measurement of damage by resorting to image correlation techniques. This full-field measurement procedure gives access to $2 \mathrm{D}$ and $3 \mathrm{D}$ displacements that can be utilized to analyze damage mechanisms, to estimate damage fields, and to determine material parameters of damage growth laws. Different features associated with image correlation are addressed in the context of continuum damage mechanics (CDM). Applications concerning damage detection, damage quantification and damage model validation are presented.
\end{abstract}

Keywords Continuum Damage Mechanics · Digital Image Correlation · Digital Volume Correlation · Microcracks · Porosities

\section{Introduction}

Before the publication of the first issue of the International Journal of Fracture Mechanics, there were only four published papers on what is known today as Continuum Damage Mechanics (CDM); one written in Russian [69], three others in English [70] 117 108]. All dealt with tertiary creep, namely, the acceleration of secondary creep due to the gradual degradation of the material. The idea of introducing a continuity variable (i.e., the complement to unity of a damage variable) was to correct for the shortcomings of secondary creep models that overpredicted the time to rupture of materials subjected to small or moderate stress levels [73]. This continuous variable was a mathematical representation of the growth of microcracks and their coalescence into a macrocrack.

Interestingly, the very first paper using damage concepts to be published in what was now the International Journal of Fracture was written by Kachanov [71] and was devoted to the analysis of crack propagation in a creeping and damaging medium. This brief communication has received very little attention (i.e., 2 citations as of 2014

François Hild · Amine Bouterf · Stéphane Roux Laboratoire de Mécanique et Technologie (LMT), ENS Cachan/CNRS/Université Paris Saclay,

61 Av. Président Wilson, 94235 Cachan, France

E-mail: \{francois.hild;amine.bouterf;stephane.roux $\} @ 1 m t . e n s-c a c h a n . f r$ 
according to the journal's site, and only one citation according to Web of Science!) in contrast with the earlier publications by the same author.

One of the main issues of this new theory was related to the fact that the continuity or damage was introduced as an internal variable field, which is not directly measurable as are, say, displacements, strains or temperatures. This observation has led to different developments, the first direction was related to what will be called physical damage in the sequel. The second route, which was to be followed in the early developments of continuum damage mechanics, is associated with mechanical damage.

The need for the evaluation of damage stems from different aspects:

- The understanding of damage mechanisms (e.g., cracking, debonding, delamination, voidage) is very useful for various reasons. For instance, the design of new materials that are more damage-tolerant [41] can benefit from the knowledge of mechanisms at play and their interaction with the underlying microstructure. Similarly, when constitutive models are developed, the understanding and possibly the quantification of the damage mechanisms has led to so-called physics-based formulations [5].

- The identification of material parameters associated with damage models is needed to predict the damage state of structures in use. This calls for robust identification procedures, in the context of noisy data, whose trustworthiness is estimated, mostly in terms of mechanical signature.

- The evaluation of the damage state of structures in use is required in the context of health monitoring (e.g., a building subjected to an earthquake, inspection of aircrafts).

The title of the present paper refers to the seminal article written by Lemaitre and Dufailly [86]. Interestingly, it was written approximately 25 years ago. It will allow us to discuss some advances that were achieved since that period. It is worth emphasizing that there are not so many papers reviewing different measurement and identification techniques applied to the evaluation of damage states. The interested reader may read the review of Maire [90] or the very recent monograph related to various aspects of CDM [147.

Eight different methods were discussed by Lemaitre and Dufailly [86]:

- The so called 'micrography' or fractography, which are usually carried out by resorting to scanning electron microscopes (SEMs). This point shows that the scale of observation is related to micrometer to sub-micrometer resolutions. Such analyses are still extensively utilized to determine damage mechanisms of materials (e.g., see Ref. [102]). Quantitative evaluations are also possible thanks to various image processing techniques developed over the years [28 35$]$.

- Density measurements are relevant to evaluate the porosity in damaged of ductile materials. By resorting to computed tomography, it is now possible to evaluate porosity distributions $[90$. It can be noted that optical and electronic micrographies can also be used to analyze fractured surfaces in terms of porosity locations and distributions.

- Ultrasonic waves and acoustic emission are non-destructive techniques that can be utilized to evaluate the damage state of materials and structures [15]52. Since 1987, tomography has become an additional tool that can be used for the same purposes as the two listed techniques 90 .

- Electrical resistance variations induced by damage can also be used to estimate the damage state for composite materials [113]2]. The equivalent technique in the 
context of fracture mechanics is the potential drop monitoring [12]. One limitation of both techniques is related to the fact that the microcracks or macrocracks are open and remain open.

- Loss of stiffness is one of the mostly used damage indicators to study various classes of materials [81 83]. The state coupling between elasticity and damage is used to describe the gradual degradation of elastic properties induced by various damage mechanisms [23].

- Cyclic stress amplitudes and their variations in fatigue experiments is another indicator of the degradation of materials in fatigue. This phenomenon is accounted for by coupling cyclic plasticity and damage [87] 82 .

- Tertiary creep has been the first damage mechanism described by resorting to a damage variable [69,117]. These early propositions have been enriched over the years by accounting for the underlying mechanisms [6 34 .

- Microhardness can also be used to quantify damage fields thanks to the change of yielding properties with damage [86, 144]. The interest is related to the fact that the material surface and sub-surface are probed locally.

All of the listed techniques are still options considered by scientists interested in one of (or all of) the three aspects of damage features listed in the third paragraph. Some have seen additional developments while others are still used as they existed 25 years ago. Conversely, some of recent experimental techniques did not exist or were in their infancy. The aim of the present paper is to discuss these newer techniques and what was made possible by using them in a CDM framework. The reader interested in the previous techniques is referred to the seminal paper of Lemaitre and Dufailly [86] and a very recent handbook on CDM [147].

In terms of visualization techniques, as already mentioned, tomography has become one tool of choice to have access to bulk information in a non destructive way since their early use in the fields of materials science [10, 133] and mechanics of materials [94]. This technique is particularly interesting when most of the damage development occurs within the bulk of the studied material (e.g., nucleation, growth and coalescence of damage in high triaxiality experiments [91]) or when damage does not occur before very late in the experiment (e.g., low triaxiality experiment [107]).

One of the quantitative uses of images consists of measuring displacement fields by resorting to different optical systems [119, 46, 120. Among these techniques, Digital Image Correlation (DIC) is more and more used, and will be the focus of the present study. It has been introduced in the early 1980's in solid mechanics [111,141,29], and has undergone numerous improvements over the years [140 60 139]. Very early on, analyses of fractured samples [101] and structures [142] were performed. One of the reasons is that the analysis of cracks is made more robust by having access to displacement fields. Another reason is due to the fact that global parameters such as stress intensity factors, crack tip opening angles can be estimated.

The previous analyses were restricted to surface measurements. On the turn of the century, Digital Volume Correlation (DVC) was introduced by Bay et al. [11]136] and applied to 3D reconstructed volumes associated with tomographic data. When studying cracked samples, crack closure [88] and crack propagation [88] have been quantified by analyzing the measured displacement fields. Similarly, numerical models have been validated thanks to direct comparisons of measured stress intensity profiles and predictions via extended finite element simulations [118]. Quantitative evaluations of damage 
are still very delicate. For instance, damage mechanisms and their quantification have been reported for particulate composites by resorting to DVC analyses [55]63].

In fracture mechanics, the identification usually consists of extracting few parameters (e.g., stress intensity factors [101, 128, energy release rates [99], parameters of cohesive zone models [1,42,130]) relying on comparisons of measured displacements with simulations or available closed-form solutions. In the context of CDM, damage is described by fields of local (or nonlocal) variables. This fact requires inversion techniques that explicitly deal with fields of unknown quantities. One approach that was specially devised to identify damage fields is the equilibrium gap method (EGM), which minimizes the equilibrium gap with respect to the relative stiffness from element to element [31, 32].

Once regularized by a chosen damage growth law, the previous case becomes easier in the sense that fewer parameters are sought. Various identification routes have been proposed [7, 46. Among them, the finite element updating technique has been the first one to use full-fields. One of the earliest applications using DIC was performed by Geers et al. [44] who determined nonlocal features for a composite material. The virtual fields method was also used to identify a damage growth law [27]. The equilibrium gap method was considered to identify a growth law that is not postulated a priori [33. Once regularized by the damage growth law and pre-conditioned, it was shown to lead to significantly finer spatial resolutions for the damage field [127, 114, 13. Last, let us note that integrated DIC approaches in which the unknown damage parameters are directly extracted from the DIC procedure have been introduced in the context of CDM by Réthoré [129].

Different aspects related to damage measurements via DIC and DVC will be exemplified in the sequel. First, the distinction between physical and mechanical damage will be explained since it has some important consequences in terms of experimental and numerical procedures. Second, the use of new imaging means (e.g., computed tomography) and their subsequent analysis with correlation techniques are introduced. Physical and mechanical damage will then be studied essentially for a given material, namely, plasterboard. This material, which is not classical, was selected because it is representative of the class of quasi brittle materials for which CDM is one tool of choice to describe their degradation. It should however not be concluded that the results reported herein are restricted to this class of materials. Other materials and mechanisms have been and still are analyzed with some of the tools introduced hereafter [90 62 57.

\section{Mechanical and physical damage}

In the above presentation, damage has different meanings depending on the point of view of the scientist who uses such a concept. This is also true when analyzing in detail the different measurement techniques proposed by Lemaitre and Dufailly [86]. To avoid any misunderstanding, two different definitions will be used, namely mechanical and physical damage. Crudely speaking, physical damage is to mechanical damage what dislocations are to plasticity. Some differences are associated with the scale at which the degradation phenomena are analyzed. Others are related to the modeling tools that will be used to describe the effect on mechanical properties.

Mechanical damage was first proposed by Kachanov [69 70] and Rabotnov [117]. Tertiary creep was introduced in which the effect of damage on secondary creep was 
included. It corresponds to a kinetic coupling between viscoplasticity and damage [87. Similarly, there exists a state coupling between elasticity and damage that leads to a stiffness loss induced by the material degradation [83. 87]. One key aspect is related to the fact that the continuity and damage were described as field variables. When coupled with elasticity, damage can only be characterized by estimating the local elastic properties of the studied material. Consequently, this description is usually limited to the largest scales. It is only remotely connected to the underlying mechanisms reducing the tangent stiffness (e.g., debonding, delamination, microcracking, microvoidage).

In the early developments of CDM, more attention was paid to predicting the component behavior rather than analyses at the scale of physical damage. Many applications and models have been developed over the years in that area [82 84. However, at one stage, this operational point of view became a liability and slowed down the widespread acceptance of CDM in the scientific community. The links with the mechanisms at play were missing or insufficiently secured [26]. The mechanisms that are related to the loss of continuity of a material are for instance the nucleation (resp. initiation), growth and coalescence of voids (resp. cracks). They are referred to as physical damage since they can be observed and quantified at lower scales than mechanical damage. Their relationship with mechanical damage is not straightforward and requires a mechanical model. For instance, Ashby and Dyson [6] have shown the relationship between a damage parameter à la Kachanov and the physical degradation in metallic materials undergoing creep. Cocks and Leckie [34 developed constitutive models accounting for such mechanisms in a CDM framework, which was subsequently used by Hall and Hayhurst [47] to predict damage growth in complex structures.

One route to link physical and mechanical damage is to resort to homogenization techniques [134,20], which allow to deal with scale changes. In particular, scale separation is desirable in this type of investigation so that a representative volume element can be constructed. Mechanical damage is defined at the macroscale, while physical damage is concerned with microscopic scales. When macroscopic gradients become important, local formulations are no longer valid and nonlocal [112] or gradient-based [110 97] damage models are proposed, for which an internal length scale naturally appears. It is worth noting that the latter is expected to grow with the damage level, an assumption that is rarely made [53,37. Furthermore, there are very few experimental studies in which this internal length is evaluated with more than a few macroscopic data.

All these theoretical developments need to be validated against experimental observations and quantifications. Of the eight above listed techniques, two categories can be distinguished with respect to physical or mechanical quantifications of damage. When mechanical damage is studied, indirect quantifications are performed by studying the changes of mechanical properties (e.g., Young's modulus, yield stress, stress amplitude), which are then coupled with damage [87. Conversely, the characterization of damage via non destructive techniques (e.g., using ultrasonic waves) allows mesocracks to be detected and possibly quantified. Computed microtomography can also be used to directly evaluate physical damage of various materials [10 $90,133.94]$.

Among the new techniques that have emerged since the last two decades, volume imaging via tomography has seen numerous results being reported [94]. This technique will also be used in the sequel with one additional feature that is less practiced, namely, volumetric displacement measurements via DVC. Its 2D counterpart, namely, DIC will also be illustrated. One of the reasons is that mechanical damage being defined as a field, full-field assessments are needed. Furthermore, the study of damage, which 
leads to strain softening, also requires full-field measurements to better understand and identify models that are able to capture localized phenomena [17].

\section{New experimental tools}

Among the previous methods of damage characterization, 3D visualization and kinematic measurement techniques were in their infancy when the paper of Lemaitre and Dufailly was published [86]. Today, they have become one of the tools of choice for damage and fracture studies. They will be quickly introduced in the two following subsections.

\subsection{Computed tomography and laminography}

3D medical imaging has revolutionized the way physicians diagnose and treat many different illnesses and pathologies. Computed tomography consists of imaging essentially elongated (or stick-like) objects by acquiring a set of radiographs for different angular positions about an axis perpendicular to the incident beam axis [66, 4]. These sets of raw data are subsequently processed to reconstruct 3D maps of X-ray attenuation coefficients [72. The X-ray sources were initially available on synchrotron beam lines, but nowadays laboratory tomographs are also used. Microtomography consists of imaging various materials at micrometer resolutions [43].

Computed microtomography has become a very powerful tool in the field of materials science [10,133,38,137,94, and more recently in mechanics of materials, in particular in the context of damage and fracture mechanics [128 61]. One key aspect is to be able to perform in situ mechanical tests [25 24]. Early studies have dealt with damage developing in particulate composites [25, 8, 9]. The three damage mechanisms of ductile materials, namely, void nucleation [75, 76], growth [18] 77] and coalescence [98 8. 133, 148] have been analyzed and quantified thanks to computed microtomography. In some instances, damage models have been compared [9] and even validated [77] with tomographic analyses. Creep has also been studied by resorting to computed tomography [116, 68, 67, 19. Similarly, microcrack initiations and propagation have been observed and quantified for particulate composites [25 55 ] and concrete samples [14] 138, 123, 63.

Ductile damage, which generally develops in the bulk of materials, can be visualized non destructively during in-situ mechanical tests. For example, the three stages of ductile damage, namely, void nucleation, growth and coalescence have been studied for dual phase steels [91. The tomography setup that was used is located at ID15 beam line of the European Synchrotron Radiation Facility (ESRF) in Grenoble, France. Figure 1 (a) shows the thresholded volume, which allows the distribution of porosities to be quantified in terms of cavity density as a function of the mean strain in the neck (see Figure 1.b)). Such quantitative observations can then be used to validate damage models [77].

Gypsum boards are sandwiches made of a core of plaster cast between two paper layers. They are finished product used in the construction industry in e.g., partition walls. The main function of the two paper layers is to carry the tensile stresses and to protect the sandwich core. The foamed core mainly contributes to the rigidity of 


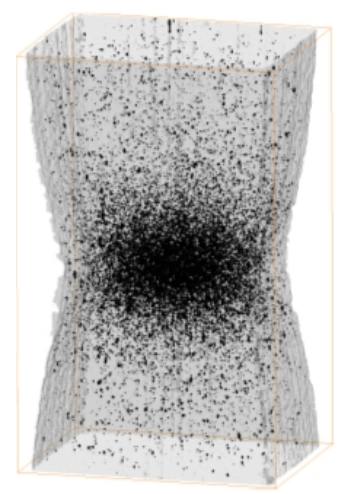

(a)

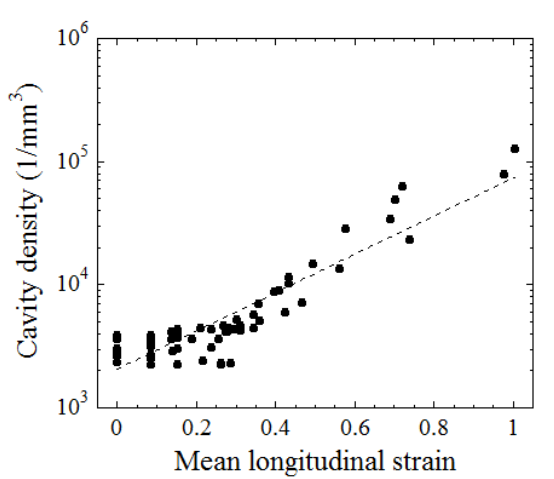

(b)

Fig. 1 (a) 3D rendering of the distribution of porosities within the deformed sample prior to fracture (courtesy of E. Maire). (b) Density of cavities as a function of the mean longitudinal strain in the neck. The solid symbols are experimental data, and the dashed line is an exponential fit

the board and to bear shear loadings. It also provides the compressive strength. Figure 2 shows a 3D rendering of a plasterboard sample subjected to three-point flexure within the lab-tomograph of LMT. The physical size of one voxel is $25 \mu \mathrm{m}$. The coarse microstructure is clearly observed in the volume of the reference configuration (Figure2(a)). Figure 2(b) shows the sample in its deformed configuration where cracks have initiated and propagated. However, their mechanical quantification needs an additional tool that will be introduced in the next subsection.

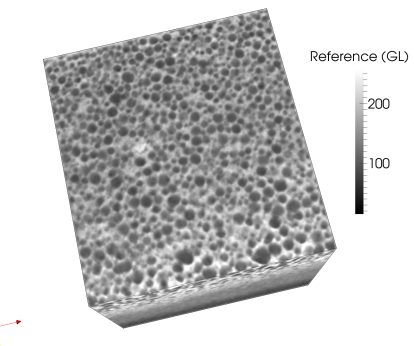

(a)

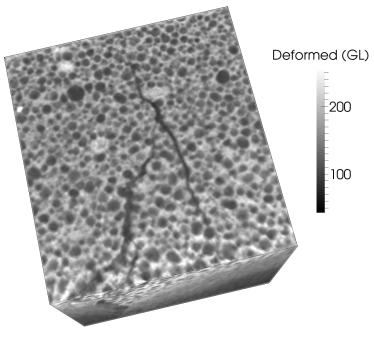

(b)

Fig. 2 (a) 3D rendering of the microstructure of foamed gypsum to be subjected to threepoint flexure. (b) The crack is visible on the lateral face of the sample

Laminography allows for the 3D imaging of elongated objects in two directions [48] 51,50,49]. Computed laminography is a generalization of computed tomography. It uses a rotation axis that is tilted by an angle less than 90 degrees with respect to the incident beam axis. Being more recent and still reserved to synchrotron beam lines, only few 
studies on damage features analyzed via laminography have been reported, namely, damage in fiber reinforced composites [103], the failure of a polymer composite [150], the development of ductile damage [106] 93, and crack initiation and propagation in aluminum alloys [135].

Laminography has been utilized to image in-situ experiments on notched samples made of aluminum alloy [104]. Figure 3, a) shows the final slanted crack by thresholding the reconstructed $3 \mathrm{D}$ volume. The same thresholded volume is shown at a very late stage of the experiment in which virtually no damage is observed (Figure 3(b)). From these observations, it may be concluded that damage developed at the very end of the experiment. However, no information on the strain state is available. The latter requires kinematic fields to be measured. This is achieved by resorting to image correlation.

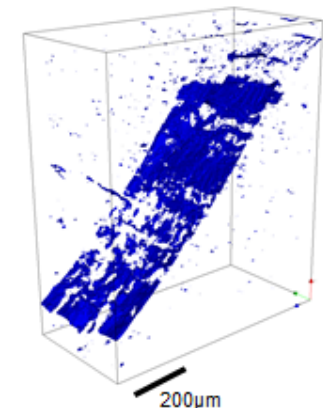

(a)

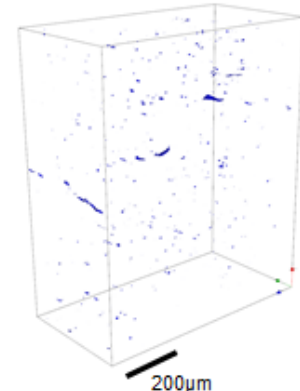

(b)

Fig. 3 Thresholded volume after (a) and prior to (b) failure of a notched sample made of aluminum alloy (see Figure 5 a)). A very late development of damage is observed 107

\subsection{Kinematic measurement via image correlation}

DIC and DVC consist of measuring displacement fields from the registration of images (or volumes) at different states of deformation [140,60, 139]. These calculations can be carried out on different types of pictures acquired by various imaging devices (e.g., atomic force microscopy, scanning electron microscopy, optical microscopy, standard cameras, computed tomography, magnetic resonance [60]). In the following, global approaches will be presented and used. It is worth noting that other approaches are available, which are referred to as local in the sense that image registration is performed on a collection of small interrogation windows in the considered region of interest [140] 139 .

One of the reasons for using global approaches is that the measured displacement fields are expressed in terms similar to kinematic bases used in numerical simulations. This feature is particularly appealing when identification and validation procedures of damage models are developed. Another reason is the fact that DIC and DVC can be regularized by mechanical properties (e.g., static equilibrium [131 79 146]). This 
type of approach allows the discretization to be very fine at no loss of measurement uncertainty $[80] 78]$.

In global approaches, the image registration aims at minimizing the sum of squared differences of the picture $f$ in the reference configuration, and $g$ in the deformed configuration corrected by the measured displacement field $\boldsymbol{u}_{m}$ over all pixels $\boldsymbol{x}$ (or voxels) belonging to the region of interest (ROI)

$$
\boldsymbol{u}_{m}=\operatorname{Argmin}_{\boldsymbol{u}} \sum_{\mathrm{ROI}}(f(\boldsymbol{x})-g(\boldsymbol{x}+\boldsymbol{u}(\boldsymbol{x})))^{2}
$$

Formulated as such, this ill-posed problem cannot be solved directly since the number of unknown degrees of freedom is greater than the number of scalar gray level conservation equations, not to mention the fact that pictures and reconstructed volumes are corrupted by various acquisition features. To regularize the minimization problem, the displacement field is decomposed over a chosen kinematic basis

$$
\boldsymbol{u}=\sum_{i} u_{i} \boldsymbol{\mu}_{i}(\boldsymbol{x})
$$

where $u_{i}$ are the unknown kinematic degrees of freedom and $\boldsymbol{\mu}_{i}$ the trial (i.e., chosen a priori) displacement fields, which span over the whole ROI (i.e., Rayleigh-Ritz approach) or over the elements connected to the considered nodal displacement (i.e., Galerkin approach). As will be shown in the sequel, the choice of the trial displacements $\boldsymbol{\mu}_{i}$ can be tailored to the application at hand, or associated with general purpose finite element kinematics (e.g., shape functions of 4-noded quadrilaterals [16], 3-noded triangles [79], 8-noded cubes [127]). For a finite element based DIC (or DVC) procedure, the size of the element $\ell$ corresponds to that of the element edge.

The overall quality of the registration is characterized by the global correlation residual $\Phi_{c}$ such that

$$
\Phi_{c}^{2}=\sum_{\mathrm{ROI}} \rho^{2}(\boldsymbol{x}),
$$

the latter being minimized (see Equation (1)). The level of the global residual at convergence can be related either to the dynamic range of the analyzed pictures [60] or the variance associated with acquisition noise [100. More importantly, a whole correlation residual field $\rho(\boldsymbol{x})=f(\boldsymbol{x})-g(\boldsymbol{x}+\boldsymbol{u}(\boldsymbol{x}))$ is available, which is computed for each pixel (or voxel) position $\boldsymbol{x}$. Any local mismatch between the assumed kinematics and the experimental one can be detected thanks to this quality estimator. This property turns out to be very useful in the context of damage [61 62 62 [] as discussed in Section 4.1

When needed, mechanical regularization can be incorporated in the minimization scheme as in any inverse problem [145]. In the present case, a mechanics-based regularization will be considered. It is based upon the equilibrium gap functional

$$
\Phi_{m}^{2}=\{\boldsymbol{u}\}^{t}[\boldsymbol{K}]^{t}[\boldsymbol{K}]\{\boldsymbol{u}\}
$$

that is expressed for an elastic medium for which all load-free nodes should have vanishing nodal forces (i.e., $[\boldsymbol{K}]\{\boldsymbol{u}\}=\{\mathbf{0}\}$ ), where $[\boldsymbol{K}]$ is the associated rectangular stiffness matrix. All inner nodes satisfy this condition when no body forces are considered. Similarly, traction-free boundaries are also included in the set of nodes to compute the rectangular stiffness matrix $[\boldsymbol{K}]$. For the boundary nodes that are not traction-free (i.e., those that correspond to Dirichlet boundary conditions), ad hoc regularizations have been proposed in $2 \mathrm{D}$ and $3 \mathrm{D}$ settings. The interested reader will find more details 
about such functionals $\Phi_{b}$ in Refs. [80 78 146 143]. The three functionals $\Phi_{c}, \Phi_{m}$ and $\Phi_{b}$ are considered in a total functional $\Phi_{t}$, which is a weighted sum

$$
\Phi_{t}\left(\boldsymbol{u}_{m}\right)=\frac{\Phi_{c}\left(\boldsymbol{u}_{m}\right)}{\Phi_{c}(\boldsymbol{v})}+\left(\frac{\ell_{m}}{\lambda}\right)^{4} \frac{\Phi_{m}\left(\boldsymbol{u}_{m}\right)}{\Phi_{m}(\boldsymbol{v})}+\left(\frac{\ell_{b}}{\lambda}\right)^{4} \frac{\Phi_{b}\left(\boldsymbol{u}_{m}\right)}{\Phi_{b}(\boldsymbol{v})}
$$

where $\boldsymbol{v}$ is a normalization displacement field, here chosen as a plane wave whose wavelength is denoted by $\lambda$. It follows that $\ell_{m}$ and $\ell_{b}$ define regularization lengths such that the optimization is controlled by DIC for length scales less than $\ell_{m}$ and $\ell_{b}$. Conversely, for length scales greater than $\ell_{m}$ and $\ell_{b}$, the displacement fluctuations that are not mechanically admissible are filtered out. These new lengths $\ell_{m}$ and $\ell_{b}$ play a similar role as the element size when DIC and DVC are not regularized. When $\ell \gg \ell_{m}, \ell_{b}$, the regularization is not active, whereas $\ell \ll \ell_{m}, \ell_{b}$ a strong regularization is enforced. Last, when $\ell \approx \ell_{m}, \ell_{b}$, a mild regularization is applied. This case allows high frequency fluctuations to be dampened while not strictly assuming an elastic behavior.

To illustrate the effect of regularization, Figure 4 shows two measurements of displacement and strain fields using an elastic regularization. The highest strain levels are clearly the manifestation of cracks or discontinuities, which cannot be resolved as a continuous displacement is sought. A large regularization length smoothes out and spreads the strain over a large region. Conversely, a lower regularization length does not damp fluctuations in the displacement field resulting in measurement uncertainties. It is noteworthy that in the present case, the displacement is decomposed over an unstructured mesh of triangular elements of edge size $\ell=3$ pixels (i.e., about $200 \mu \mathrm{m}$ ). Without regularization, a plain DIC algorithm would not converge. This case will be revisited in Section 5.2 in which the mechanical regularization will be relaxed in the cracked zones via a damage model.

To exemplify one possible application of DVC, which is unregularized in the present case (i.e., $\ell_{m}, \ell_{b}=0$ ), Figure 5(a) shows the analyzed microstructure and the corresponding region of interest (see Figure 3 for two deformed configurations). This is a very difficult texture for DVC purposes since the content of secondary particles is less than $0.5 \%$. Even under such extreme conditions [105], the strain field has been evaluated once the displacement field has been measured. Figure 5(b) shows von Mises' equivalent strain field corresponding to the deformed configuration of Figure 3 (b). From these two complementary pieces of information, namely, virtually no damage (Figure 3(b)) and very localized strains (Figure 5(b)), it could be concluded that the slanted shape of the crack is due to localized plastic strains and that damage develops very late in the experiment on the tested aluminum alloy [107.

\section{Physical damage}

As above discussed, "physical damage" refers to the elementary features that are responsible for a degradation of the mechanical properties. Most frequently, these mechanisms correspond to microcracks or voids. Focusing on physical damage means that the aim of an experimental characterization would be to capture these features individually.

Although it may appear as a well-defined goal, for many materials looking for these mechanisms has to be carried out at a microscopic scale (e.g., cavities for ductile damage in metals and alloys may be well below $1 \mu \mathrm{m}$ in size, damage in elastomers containing fillers may occur through debonding at scales of the order of 1-50 nm [151]), or may induce a displacement discontinuity (i.e., crack opening) that is much smaller 


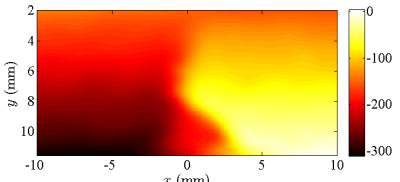

(a) $\ell_{m}=\ell_{b}=50$ pixels

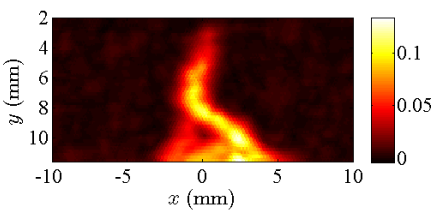

(c) $\ell_{m}=\ell_{b}=50$ pixels

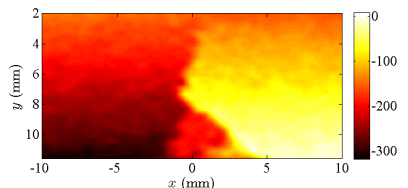

(b) $\ell_{m}=\ell_{b}=20$ pixels

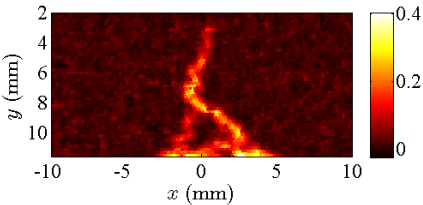

(d) $\ell_{m}=\ell_{b}=20$ pixels

Fig. 4 Zoom over a damaged region under the central point load $(x=0)$ in a three-point flexural test on a plasterboard sample. The horizontal component of the displacement field (expressed in $\mu \mathrm{m}$ ) is shown on the top when two regularization lengths are chosen. The lower set of subfigures shows maps of the major principal strain when the same regularization lengths as above are considered. The pixel size is $71 \mu \mathrm{m}$

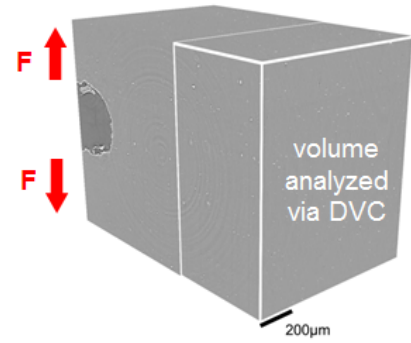

(a)

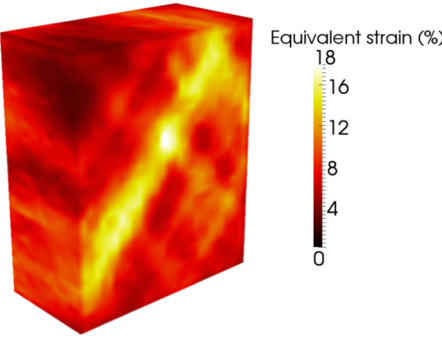

(b)

Fig. 5 (a) 3D rendering of the notched sample in its reference configuration and the corresponding region of interest, which is shown in Figure 3 in two deformed configurations. (b) Von Mises' equivalent strain field for the deformed configuration shown in Figure 3 (b)

than the crack extension. Thus the question of spatial resolution is absolutely crucial for this part. For instance, the advent of high resolution tomography has recently pushed the limit of detection of plastic cavities down to tens of nanometers, showing that previously analyzed nucleation and growth statistics were markedly limited by the spatial resolution of the imaging technique [149].

As mentioned in Section 1, direct observation techniques have always been exploited to resolve the origin(s) of mechanical degradation. Imaging techniques have soon played a major role in this quest because of the small scale of defects they could reveal. Above 
all, X-ray tomography, because of the three-dimensional character of images and the nondestructive nature of the imaging technique has revealed extraordinarily powerful. Over time, the development of lab-scale tomographs rather than synchrotron-based facilities has made microtomography more and more popular for physical damage detection and visualization. The ever increasing sensitivity and resolution of all imaging techniques has allowed for a considerable progress. Similarly, high-speed tomography is developed so that following the development of damage through time (or loading) becomes accessible even for situations that used to be challenging such as viscoplasticity 92.115$]$.

In spite of this outstanding progress, the question of the possible relevance of unresolved details always remains present, motivating the quest for yet higher resolutions. In this context, DIC (or DVC alike), may shed some additional light on two points:

- First, DIC residual fields enable for the visualization of features that are present but difficult or impossible to isolate from a single image (e.g., Figure 2 .

- Second, in addition to the "traditional" detection of defects, the analysis of the displacement field surrounding an existing defect may help qualifying its severity, allowing one to sort the identified features according to their mechanical "expression" rather than their morphological characteristics. The correspondence between these two measurements of severity is often straightforward for simple shapes (e.g., spherical cavity, penny-shaped crack) and when their interactions can be neglected. However for complex features (such as cracks emanating from odd shape pores, branching cracks, or interface cracks) the evaluation of the severity of damage is a much more difficult issue, for which DIC may provide novel tools.

Therefore, DIC can be seen as a sensitivity-enhancer for imaging techniques, giving access to additional pieces of information. The above two items are now discussed in more details in the following subsections.

\subsection{Detecting defects from DIC residuals}

DIC residual fields $\rho(\boldsymbol{x})$ reveal essentially where image registration fails. There may be a variety of reasons why DIC is not perfect, but one is that the ill-posedness of the determination of the displacement field requires a regularization of some sort that cannot do justice to any arbitrary form of displacement. In particular a discontinuity is a severe lack of regularity that is typically unexpected in most of the region of interest. Thus it is generally rewarding to assume that the displacement field is continuous [59]. When it is not, not only is the measurement inaccurate (as some compromise has to be found), but the residuals are expected to depart from the otherwise expected noise characteristics. Typically the residuals are expected to highlight specifically discontinuities when they exist. Figure 6 shows an example of a complex crack pattern in plasterboard subjected to three-point flexure, which is almost impossible to detect from the raw images even though the sample was not speckled with black and white paint.

This observation is not a guarantee for the detection of any defect. For instance, as a crack opening vanishes, so do the residuals. However, typically a 0.1 pixel opening is a standard detectability limit (or resolution), well below what can be expected from traditional image analysis treatment. Moreover because it is an image difference, its 


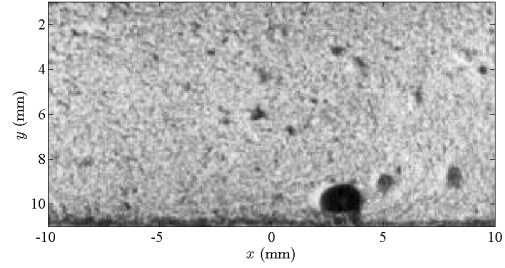

(a)

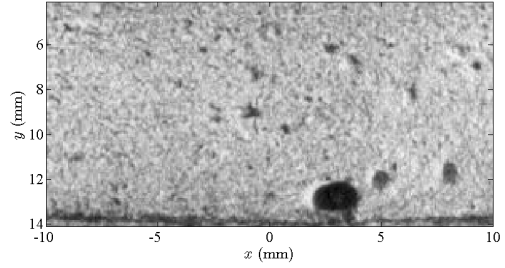

(b)

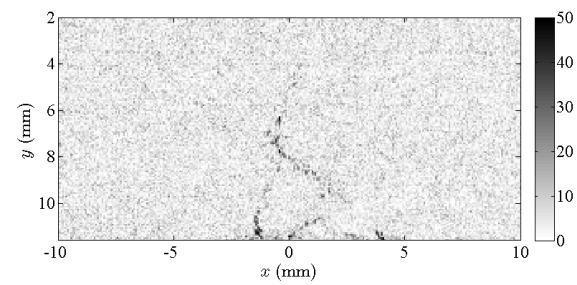

(c)

Fig. 6 Illustration of the gray level reference (a) and deformed (b) images, as well as the gray level difference (c) after registration. As most features of the microstructure are encountered in both images and as the image registration technique has brought them on top of each other, the residual image displays essentially the remaining noise, or any violation to an underlying assumption on the kinematics (i.e., cracks). Comparison with Figure 4 (c-d) confirms that the residuals reveal cracks

spatial resolution is at the pixel size, namely, corrugations of a crack path meandering through a fibre bundle can ideally be resolved pixel-wise [57].

This property is fairly general and also holds for three dimensional images. To illustrate this message, a three-point flexural test on a plasterboard specimen performed within a lab tomograph is considered. 3D Images of the reference and the damaged sample are shown in Figure 2. The voxel size was chosen to be $25 \mu \mathrm{m}$. DVC was performed over a volume of $344 \times 376 \times 344$ voxels or $8.6 \times 9.4 \times 8.6 \mathrm{~mm}^{3}$, with a regular mesh consisting of 8 noded (i.e., C8) elements of size $\ell=8$ voxels or $0.2 \mathrm{~mm}$. Regularization lengths $\ell_{m}=\ell_{b}=16$ voxels were selected. Figure 7(a) shows the residuals where the $\lambda$-shaped crack is clearly apparent. In this figure, the lower residual values have been made transparent to offer a $3 \mathrm{D}$ view on the voxels bearing the higher values. As noted in Figure 2, the crack was already quite visible on the deformed volume. Thus in order to show the benefit of working on residuals, Figure $7 \mathrm{~b}$ ) shows a similar 3D rendering of the deformed image in which the gray levels corresponding to the crack and air are very close, thereby making it more difficult to fully detect the crack.

\subsection{Qualifying damage severity}

Once a defect has been identified, it is essential to evaluate its possible influence on the mechanical behavior of the material element in which it is embedded, or its "severity." 


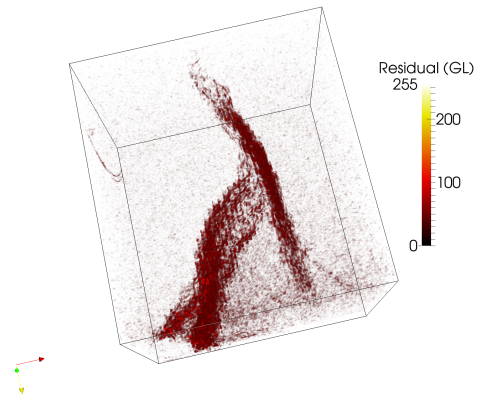

(a)

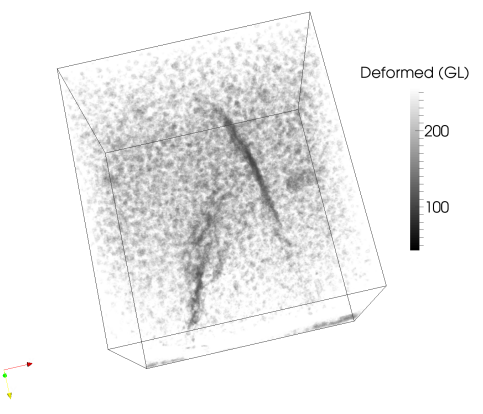

(b)

Fig. 7 (a) Illustration of gray level differences after the registration of two 3D tomographic images. The small residual values have been made transparent to render large values visible, and hence to reveal the presence of a branched crack in plasterboard subjected to 3-point flexure. (b) The same procedure is applied to the deformed image. It is more difficult to detect the extent of the cracking pattern

Within an infinite elastic medium, the influence of a localized defect (i.e., the variation of stress or strain due to its presence for a uniform stress or strain at infinity) can be written as an infinite discrete sum of elementary fields decaying with the distance $r$ to the center of the defect as $r^{-d-n}$ where $d$ is the space dimension, and $n \geq 0$ is a positive integer index [40]. At a large distance from the defect, only the $n=0$ terms matter. These fields correspond exactly to exterior Eshelby solutions.

In two (resp., three) dimensions, there are three (resp., six) such functions, which correspond to a local change of volume for one term, and uniform shear for the others. Let us stress that it is not needed to assume that the defect is a uniform change in elastic properties within an ellipsoidal inclusion. If this is the case, then the amplitudes of all terms of indices $n \geq 1$ are identically null. Otherwise, even for a defect of arbitrarily complex shape, the same dominant fields can be used, and hence the three (or six) scalar amplitudes, $a_{i}$, characterize the defect (or an equivalent one at large distance). Dimensionally, the external Eshelby fields can be chosen conventionally outside a disk (or sphere) of radius $R$ because these fields are self-similar. The amplitudes $a_{i}$ are proportional to $R^{-d}$. The dependence over the distance being set, the only remaining quantity is the linear relationship between the stress at infinity and the local stress variation considered at scale $R$. The link between the two defines a $4^{\text {th }}$ order tensor, which has been introduced in Eshelby's solution (and is now termed Eshelby's tensor).

Let us stress the fact that the influence function of a local zone extends to infinity. Large distances as such are of little interest because of the rapid decay of Eshelby's solution with distance, $r^{-d}$, yet there is a region of space surrounding a defect where its influence can be probed. This gives an extra sensitivity as compared to a simple morphological approach in defect detection.

In the above discussion, one single defect has been considered within an infinite homogeneous matrix, ignoring possible interactions. This may not be as dramatic as one could fear. As a matter of fact, most (if not all) homogenization techniques are based on a perturbative analysis where the interactions are not directly taken into account but only through an effective medium around the elementary constituents [134]. These 
approaches have been refined to sophisticated treatments yet the philosophy remains mostly unaltered. The here proposed treatment of defect severity proceeds from the same lines of thought. The broad success of these homogenization theories suggests that ignoring local interactions but concentrating on global collective effects already provides a very powerful framework. Key to this success is the ability to capture the mean effective behavior of the surrounding medium. It comes into play in Eshelby's problem through the property of the embedding matrix.

It is noteworthy that when considering the joint effect of two defects, at a distance larger than their relative separation, again the perturbation of the stress and strain field can be cast in the same framework of Eshelby-type outer field. When the interactions can be neglected (e.g., when the defect sizes are much smaller than their separation) then the global amplitude is nothing but the sum of the two individual amplitudes. Otherwise, interactions will induce a breakdown of the additivity rule. This implies in particular that possible spatial correlations do matter in the severity of defects, in a similar way as spatial correlations have an impact on macroscopic effective properties 134.

Severity quantified from the Eshelby amplitudes is also a way to define an equivalent defect that may be introduced for modeling convenience. According to the previous discussion, there is always an elastic ellipsoidal inclusion that will have a similar radiated far field. Size, shape and amplitude may each be modified provided the resulting global amplitude is kept fixed.

Because the far field radiated by a defect has a well-defined shape, it is possible to use this knowledge to characterize the defect severity from the amplitude of the corresponding Eshelby's field. Formulated this way, the problem becomes easily solvable with a global DIC approach. Either the displacement field has been first measured and severity has to be estimated from a post-processing step of this first result or a novel (refined) DIC analysis is considered. Although they look like rather different approaches, in theory, similar results are to be expected under some specific conditions [100]:

- first, the discretization errors should be comparable in both cases. It is unfair to use a coarse mesh for the prior measurement of the displacement and an analytic description of the displacement at the pixel level for the second analysis.

- second, not only are the displacement measurements of interest but also their uncertainties (which differ from one degree of freedom to the next), and their mutual correlations [59]. Ideally the full covariance matrix of all pairs of degree of freedom used in the description is to be used through a metric (norm) to measure the discrepancy between measurement and expectation [45, 100.

As soon as one (at least) of these two conditions is not satisfied, there is a net benefit to resort to a new (enriched) DIC analysis.

The above discussion assumed that a single "brittle" microcrack was nucleated (and stopped) in a linear elastic matrix. Let us first note that the close vicinity to the defect could involve a much more complicated behavior than just the occurrence of a microcrack. Local plastic flow may occur together with a microcrack, or the defect may simply be the ductile expansion of a cavity. Away from the defect, in the surrounding elastic medium, there is no way to distinguish a loss of rigidity from a plastic strain at a given load using the exterior Eshelby amplitude. However, if the medium is analyzed 
at two different load levels, then the distinction becomes clear by considering that the amplitude has an affine dependence on the load. The constant part can be attributed to plastic strain whereas the linear part is relative to damage.

If the surrounding medium is in a homogeneous state well inside the plastic domain, linearization of the response is always an option. However, it is to be underlined that one difficulty of the problem is that the tangent elastic behavior will be different if the medium is further loaded or unloaded. For a single defect in an elastic medium, in the very simple case of an anti-plane 2D setting, this problem can actually be solved [124]. It turns out that the range of interaction is variable and the algebraic decay of the stress perturbation takes place with an exponent that depends on the loading to unloading moduli ratio. This can be seen as a generalization of Eshelby's outer solution, which however lacks the wide applicability of Eshelby's solution because of breakdown of the superposition property of elastic solutions in those sign-dependent tangent moduli. What remains of wide applicability is the fact that suspecting the presence of a defect in a medium whose behavior and state are well known, it is always possible, at least numerically, to compute the sensitivity field due to the presence of a localized defect. A scale separation between the defect size and the scale over which the tangent elastic moduli can be computed allows one to provide an influence function equivalent to the Eshelby amplitude that is a different measure of the defect severity.

\subsection{One-dimensional medium}

The previous discussion also holds in a one-dimensional medium described say as an Euler-Bernoulli beam to mention one of the simplest examples. However, the 1D geometry gives a much broader range of influence for defects. The perturbation due to local damage rather than vanishing away from the defect can now be felt at arbitrarily large distances, and judged from displacements, the influence function diverges at infinity. Thus it is more convenient to compute the influence functions (expressed either in terms of displacements) for a beam of finite length, and specified boundary conditions. (Previously, for a 2D or 3D infinite medium, the influence of a defect decays with the distance as $r^{-d}$ for stress and strains and $r^{1-d}$ for displacements, and hence using a constant stress or strain at infinity made no difference, and boundary conditions (when sufficiently remote) could be ignored.) For beams, because boundary conditions matter, the influence functions are no longer translationally invariant and have to be computed for each possible position of the defect.

Longitudinal and transverse displacement components are essentially decoupled (although a no-through crack breaking down the axial symmetry of the beam will induce a small coupling). First, for the longitudinal load (along the $x$ axis), the considered boundary conditions are a prescribed displacement at both ends of the beam, $x=0$ and $x=L$. A zone that contains a defect $x_{c} \leq x<x_{c}+\ell$ will give rise to an additional displacement field that assumes the following form

$$
G_{x}\left(x ; x_{c}, \ell\right)=\left\{\begin{array} { l } 
{ - x / ( L - \ell ) } \\
{ ( x - x _ { c } ) / \ell - U x _ { c } / ( L - \ell ) } \\
{ 1 - ( x - \ell ) / ( L - \ell ) }
\end{array} \quad \text { for } \quad \left\{\begin{array}{r}
x<x_{c} \\
x_{c} \leq x<x_{c}+\ell \\
x_{c}+\ell \leq x
\end{array}\right.\right.
$$

When $\ell$ is small as compared to the beam length, this influence function resumes to a simple step function with a unit discontinuity located at $x_{c}+\ell / 2$. Focusing on the damaged zone, the potentially complicated displacement field is turned into a uniform 
strain $1 / \ell$. The analogy with Eshelby's problem is emphasized although the $1 \mathrm{D}$ axial case looks trivial. At a large distance from the defect, there is no way to qualify the defect by anything else but the resulting displacement jump.

For transverse displacements, the philosophy is quite similar, although the influence function is different. The considered boundary conditions are again a prescribed transverse displacement $u_{y}$ and rotation $d u_{y} / d x$ at both ends, $x=0$ and $x=L$. The zone that contains the defect is described as a small region of constant curvature. Thus the influence function reads

$$
\begin{aligned}
& G_{y}\left(x ; x_{c}, \ell\right)= \\
& \left\{\begin{array}{l}
x^{2} /(L-\ell)-a(x / L)^{2}(3-2 x / L) \\
x_{c}\left(2 x-x_{c}\right) /(L-\ell)-\left(x-x_{c}\right)^{2} / \ell-a(x / L)^{2}(3-2 x / L) \\
\left((x-\ell)^{2}+2 \ell x_{c}\right) /(L-\ell)-\left(2 x-2 x_{c}-\ell\right)-a(x / L)^{2}(3-2 x / L)
\end{array}\right.
\end{aligned}
$$

$$
\text { for }\left\{\begin{aligned}
& x<x_{c} \\
& x_{c} \leq x<x_{c}+\ell \\
& x_{c}+\ell \leq x
\end{aligned}\right.
$$

where $a=\left(2 \ell x_{c}\right) /(L-\ell)+\left(2 x_{c}-L\right)$. Figure 8 shows the influence functions for axial (a) and transverse (b) displacements.
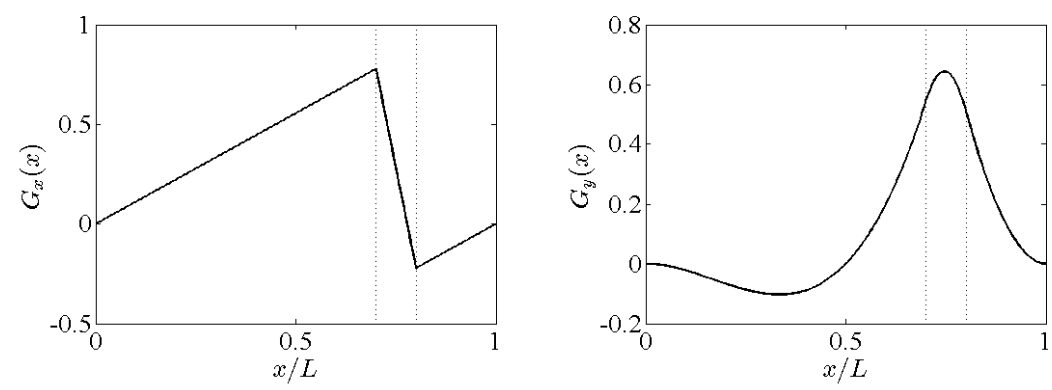

Fig. 8 Influence functions for a defect located in the region $0.7 \leq x / L<0.8$ shown with dashed lines. (a) Axial displacement, (b) transverse displacement components

Based on the above expression of the influence function, the "severity measurement toolkit" is now clear. For the axial case, no defect means that a uniform strain is expected, and hence the sought displacement field is first described as linear. In all cases, it is recommended to account for an imperfect clamping condition, and to consider the possibility of a translation. Thus, the kinematic basis consists of $\mu_{x}^{1}(x)=1$, $\mu_{x}^{2}(x)=x$. The presence of a defect at position $x_{c}$ can be probed by a third kinematic field $\mu_{x}^{3}(x)=G_{x}\left(x ; x_{c}, \ell\right)$. Hence, a severity analysis for an axial loading would consist of a three degree of freedom DIC analysis. Along the transverse direction, the procedure is quite similar, namely, for a beam (or plate) that bears no load along its length, four degrees of freedom are needed to describe its transverse displacement. For a three-point flexural test, the central load requires a fifth degree of freedom. Last, 
the influence function for a damaged zone is a sixth degree of freedom. Neglecting axial damage, but accounting for a possible (spurious) axial loading, means that eight degrees of freedom have to be taken into account. The analytic expression of these displacement fields being known, not only in the language of beam theory along the neutral axis, but thanks to Euler-Bernoulli assumption in the entire 2D side view, a DIC procedure can easily be written down.

To illustrate this procedure, the severity of the complex crack pattern observed in the three-point flexural case shown in Figures 4 and 6 is quantified. A single interval of width $\ell$ centered about the central loading point is chosen. Because of the three-point flexural geometry, most of the damage occurs there up to late stages of loading. The analysis of the severity of damage has been studied with a specific DIC procedure that describes a three-point flexural test (including an arbitrary rigid body motion) within an Euler-Bernoulli framework plus the above described influence function (only for the transverse motion since no axial loading is applied). This procedure is run on the first 15 pictures shot at increasing deflection. (On the 16th picture, a secondary crack on the side appears, and it would require a specific treatment to take it into account.) Figure 9 shows the deflection and section rotation of the plasterboard sample.

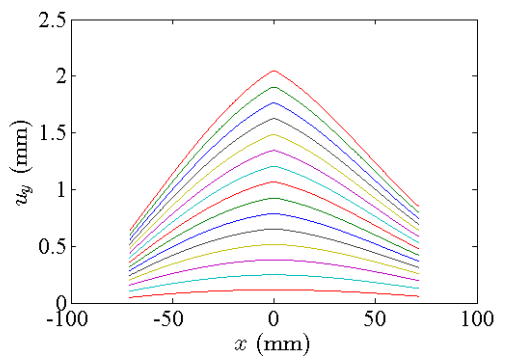

(a)

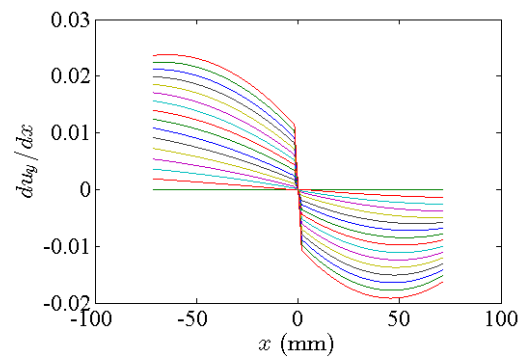

(b)

Fig. 9 (a) Deflection and (b) rotation analyzed with a DIC procedure based on an EulerBernoulli description enriched with the deflection severity function $G_{y}$, centered about the central contact point, $x=0$ and having a width of $\ell=100$ pixels, or $7.14 \mathrm{~mm}$.

The "severity" $s$ of the damaged zone is now quantified by considering the curvature within the central area $\kappa$ as compared to the extrapolation of the curvature $\kappa^{e}$ at $x=0$ from the undamaged region. As previously noted, this severity is expected to depend on the chosen size $\ell, s(\ell)$. One possible representation of the same defect is to substitute to the damaged region a homogeneous zone where the flexural stiffness has been reduced by a $(1-D)$ factor. This is precisely what is assumed when dealing with a damage law so that $D$ would be read as a damage parameter. The continuity of the flexural moment, and the assumption that outside of the analyzed zone no damage is present implies that the severity is related to $D$ through

$$
s=1 /(1-D)
$$

The observation that the severity is scale-dependent has the consequence that damage is also scale-dependent. As above mentioned for the Eshelby influence function, the 
amplitude of severity depends on the scale of the "inclusion" over which damage is spread. Yet there exists an invariant, which is the outer Eshelby amplitude. The same property holds for the plasterboard specimen. The integral of the curvature over the "damaged" zone provides the slope discontinuity over the zone. Hence, $\kappa \ell$ is the slope discontinuity, $\Delta \theta$ plus the undamaged curvature over the same interval $\kappa^{e} \ell$, and hence

$$
\Delta \theta=(s-1) \kappa^{e} \ell
$$

so that the scaled severity $(s-1) \ell$ should be an $\ell$-independent quantity. To illustrate this point, the severity analysis is computed with the above described procedure with a larger zone $\ell=200$ pixels. As shown in Figure 10(a), the severity is dependent on length $\ell$, namely, a smaller zone exhibits a larger severity. Figure 10 (b) shows that the scaled severity $(s-1) \ell$ has an identical behavior for both lengths $\ell$ although the two DIC analyses were totally independent.

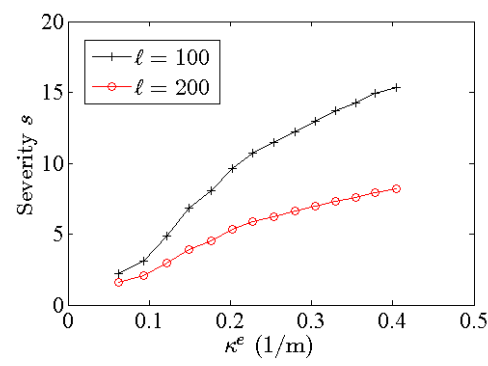

(a)

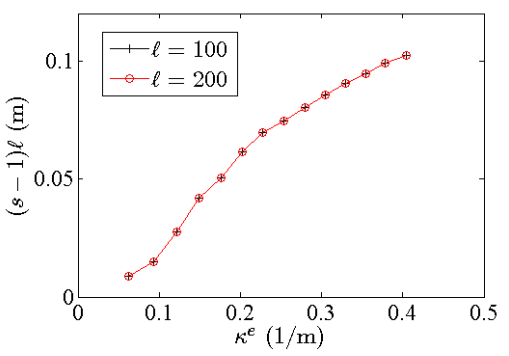

(b)

Fig. 10 (a) Raw and (b) scaled severity for the two chosen sizes of the damaged region, $\ell=100$ and 200 pixels. (a) The measured severity is dependent on $\ell$. (b) The rescaled severity is length-independent

The details of the concentration of curvature are not essential as far as the ambition is not to describe exactly the defect, but rather to capture its global severity. This observation leads to the property that damage occurring in an interval of length $\ell$ can be "lumped" at a single point [30 305 96]. This corresponds to increasing the local curvature while reducing the damaged zone size, so that the integral of the curvature, i.e., the difference in the deflection angle $\Delta \theta$ remains constant. The damage law defined at a given scale $\ell$ thus can be translated into this language as

$$
\Delta \theta / \kappa^{e}=\frac{\ell D}{(1-D)}
$$

Rather than dealing with a uniform damage spread over the interval where the curvature would be affected, the lumped damage point of view is to account for the same effect at long distances through a rotation discontinuity for the beam. This argument developed for the transverse displacement, also holds for the axial component. In that case, as $\ell$ tends to 0 , the influence function involves a discontinuity in $u_{x}$. Although 
one could discuss about the local fidelity of the defect picture, it has a tremendous advantage in terms of simplicity, and yet it may capture damage severity very accurately and provide a fair global mechanical description.

It is also gratifying to observe that the lumped damage approach can easily be recast into the DIC formalism. The three-point flexural test then consists of two beam elements joined at the center contact, $x=0$. For the axial displacement, an ideal test would suggest the continuity of axial displacement and strain. For the transverse displacement, the continuity of the displacement is anticipated, whereas the lumped approach frees a rotation discontinuity. In terms of DIC implementation, it is rather straightforward to use two beam elements (each consisting of a 6 degree-of-freedom element, two displacement component and a rotation at each end), and possible continuities are set using Lagrange multipliers.

For the very same three-point flexural test, this approach was followed and the results are shown in Figure 11 The transverse displacements (a) and rotations (b) are in excellent agreement with the previous determination based on the severity measurement, apart from the cusp, which is occurring at the central contact point in contrast with the uniform curvature imposed in the severity measurement. The rotation discontinuity $\Delta \theta$ is directly estimated as one of the kinematic amplitudes of the DIC analysis. The curvature at the central point, $\kappa^{e}$ is evaluated from the common value of the left or right limit. (Actually these two values differ by a small amount, although they should be equal in an ideal test.) The $\Delta \theta$ vs. $\kappa^{e}$ plot (Figure 11.(c)) provides the "lumped estimate" of the defect severity as a function of the loading at this point, i.e., the extrapolated curvature that stands for the flexural moment as the specimen is considered as undamaged. Figure 11.d) translates this severity into a damage parameter $D$ for a conventionally chosen size of the damaged region, here $\ell=100$ pixels, where the horizontal axis has been chosen to be the curvature in the damaged region $\kappa=\kappa^{e} /(1-D)$.

Let us come back to the case of isolated defects where the above approach is the most appropriate. The design of DIC using the above listed eight fields is called Integrated DIC (I-DIC), as the analytical form of all basis functions is derived from the mechanical analysis of the problem [58] 125. Because these functions share the same support they are not naturally independent of each other. However, they can be made orthogonal with respect to DIC. Let us recall the expression of the DIC matrix for the horizontal component of the displacement field [60.

$$
M_{i j}=\iint\left(f_{, x} \mu_{x}^{i}\right)\left(f_{, x} \mu_{x}^{j}\right) \mathrm{d}^{2} \boldsymbol{x}
$$

When the image texture is well sampled by the different basis functions, a mean-field approximation of the matrix can be used

$$
M_{i j} \approx\left\langle f_{, x}^{2}\right\rangle \iint\left(\mu_{x}^{i} \mu_{x}^{j}\right) \mathrm{d}^{2} \boldsymbol{x}
$$

Orthogonalization within this approximation is easy. It consists of subtracting to $\mu_{x}^{1}$ its mean value, and to $\mu_{x}^{2}$ its mean displacement and strain components. If scale separation cannot be used to isolate $f_{, x}$ from the basis functions, Gram-Schmidt orthogonalization can be used to diagonalize this $[\mathbf{M}]$ matrix exactly. The resulting basis is denoted by $\Psi_{x}^{i}$ for $i=1,2$ or 3 . The interesting aspect of this process is that the influence function of the defect remains associated with $\Psi_{x}^{3}$, but it is now independent of the 


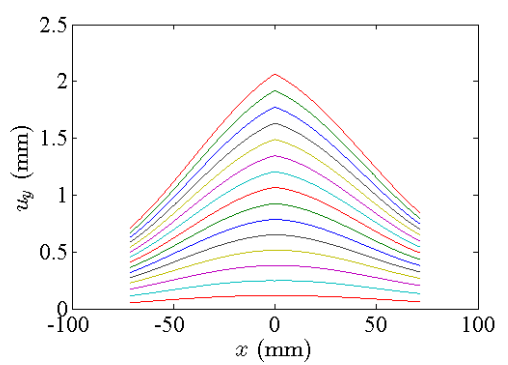

(a)

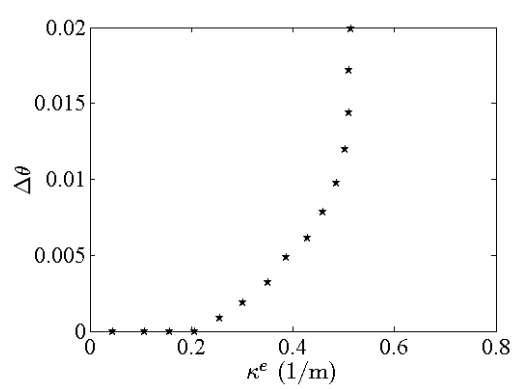

(c)

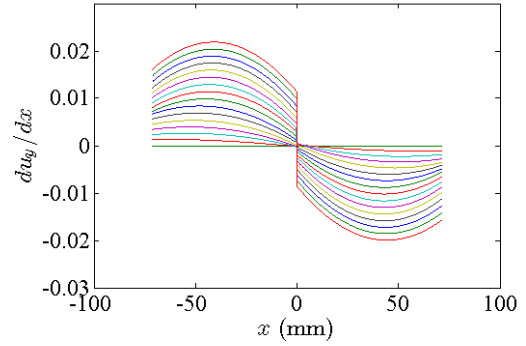

(b)

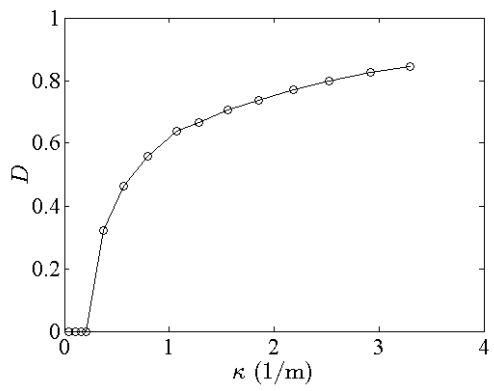

(d)

Fig. 11 Deflection (a) and rotation (b) fields obtained from a lumped damage description in a DIC analysis. (c) Resulting ratio of rotation discontinuity over curvature as a function of the curvature. (d) Conventionally choosing a length $\ell=100$ pixels, an equivalent damage $D$ is computed

mean translation and axial stretch, so that any affine motion has no impact at all on the measurement of the severity of damage.

As an additional benefit, the variance of the severity measurement, $s_{s e v}^{2}$, due to image noise is easily accessible. In the mean-field approximation, $s_{s e v}^{2}$ is inversely proportional to the noise variance, to the mean horizontal gradient $f_{, x}^{2}$, and to the $L^{2}$ norm of $\Psi_{x}^{3}$. Without approximation, the variance $s_{\text {sev }}^{2}$ is inversely proportional to the quadratic norm of $f_{, x} \Psi_{x}^{3}(x)$ [59]. The strong result that can be obtained is that amongst the different displacement functions that could capture the defect severity (actually there is an infinity thereof), $\Psi_{x}^{3}(x)$ is the one that minimizes the sensitivity to noise and that is unbiased. The very reason for this superior behavior is that it incorporates all the information available for the measurement and makes a proper use of it according to its "value" (inversely proportional to its uncertainty).

If the displacement field had been measured first, it would still be possible to measure the defect severity along similar lines from a projection of the displacement onto $\Phi_{3}(x)$. However, in order to obtain a similar quality as from I-DIC, the displacement field should have been estimated together with the covariance matrix of all kinematic degrees of freedom, and it is the latter (inverse covariance matrix) that defines the metric with which the projection is to be performed [45. 100]. 
If the expected amplitude of the $\Psi^{3}(x)$ field is small (the criterion is that the DIC cost function should not be distinguished from its parabolic approximation as the amplitude of this field is sent to 0 ) one may proceed in two steps. First, the displacement $u^{0}$ is estimated as if no defect were present, namely using $\mu^{1}$ and $\mu^{2}$ only. In a second step, in addition to the displacement field assumed to be known and now frozen, the remaining degree of freedom is evaluated from a simple additional DIC analysis using a single degree of freedom. This analysis is extremely simple, and requires a very low computational effort. Thus, it can be used to extract some additional information. For instance, one may wish to locate more precisely the position of the defect $x_{c}$ or its size $\ell$. Such a procedure was introduced in Ref. [132] to automatically capture microcracks in a uniaxial fatigue test.

Moreover, for an influence function that is translationally invariant, a fast Fourier transform reveals to be a powerful technique to speed up the analysis. However for the beam-like (1D) geometry considered herewith, the dependence of $G$ with respect to $x_{c}$ makes the analysis a little more cumbersome, but it can nevertheless be sped up significantly as compared to a straightforward implementation.

The next section on "mechanical damage" will call for such a characterization, but the chosen continuum mechanics framework will allow for considering defect interactions and collective effects more naturally.

\section{Mechanical damage}

After having seen how DIC could be used to detect defects and qualify their severity, the present section addresses the continuum mechanics description that is perfectly suited to catch collective effects resulting from the build up of spatial correlations in defect initiation and growth [82]. The previous analysis where defects were essentially embedded in an intact (homogeneous) material was rather designed to be operative for isolated defects, relevant for damage initiation(s).

The philosophy of damage mechanics proceeds somehow along a similar line of thought as previously, namely, mechanical degradation can take different forms at the local level. This is a scale where the material microstructure is to express itself. Grains, interfaces, different phases, processing defects, will speak out, and the talent of material manufacturers will make use of this variety to tailor damage resistance, strength, or ductility at will. However, when observed at a distance, a material element containing a collection of microcracks will first be seen as having a reduced stiffness [23]. Thus it can be replaced by an equivalent element having the same "severity." Choosing among all elements having the same severity, the one where the elastic properties are uniform allows damage $D$ to be defined from the relative loss of stiffness as compared to the initial state [85] 82 ].

It is to be observed that from its very foundation, damage can only be operational provided the degradation of the mechanical properties occurs in a stable fashion, evenly spread over the material elements. This stability may be broken at a macroscopic scale as captured by a localization criterion $\operatorname{such}$ as $\operatorname{det}(\boldsymbol{n} \cdot \boldsymbol{H} \cdot \boldsymbol{n})=0$ [17 56 39], a criterion that allows for a macroscopic flow of energy from the structural scale down to a plane of normal $\boldsymbol{n}$ for growing a macroscopic defect [121, 122, 21, $\boldsymbol{H}$ denotes the fourth order tangent operator. But stability can also be broken from the nucleation of a defect (e.g., microcrack, microvoid) that cannot be arrested and yet whose growth was essentially controlled from a local environment (with no need for large scale energy flow). In the 
latter case, the late stage of damage will appear as brittle and a critical damage level (much smaller than unity) will have to be introduced as a limit. Note that the latter situation may also come together with a lack of self-averaging, and hence systematic size effects and statistical variability up to macroscopic scales, akin to brittle solids.

Those stages of unstable failure, cracking or breakdown are important for reliability assessments, but by essence once started this unstable growth cannot be arrested, and hence its full characterization is not necessarily a critical issue. In contrast, the early (in the sense of stable) stages are very informative, to identify a damage law and detect foresigns of macroscopic breakdown (e.g., ruled by a localization criterion) as it can (in favorable cases) be perceived from heterogeneities developing at micro- or meso-scales.

\subsection{Nonlinear mechanical regularization}

Section 3 has shown how an elastic regularization could be used as a mechanical filter to help DIC and to produce a smooth displacement field yet preserving fidelity with respect to data. Two points of view can be adopted to justify this technique. First, in the spirit of applied mathematics, regularization is an alternative to a specific discretization where rather than choosing specific finite element shape functions, the local displacement field has to remain close to the kernel of a differential operator [89. Then, prescribing a linear operator and asking for neutrality with respect to rigid body motions and uniform strains leads naturally to the form used in Section 3.2 In such a case a piecewise linear shape function is not more (nor less) legitimate than the kernel of an operator. Second, giving precedence to mechanics, elastic regularization can be seen as a way to impose locally a homogeneous linear elastic behavior. However, in all cases, as the assumed elastic behavior becomes closer to the actual behavior of the material under study, regularization bias will be reduced, namely, the more faithful to reality, the better it is!

Therefore, as one moves toward nonlinear responses, it is natural to use in the regularization a better account for the constitutive law. Formally, the regularization form is simply a penalty to the equilibrium gap, $\|\operatorname{div}(\boldsymbol{\sigma})\|^{2}$ (in the absence of body forces) or equivalently on its discretized form (see Equation (4)). If stresses are related to strains (and hence displacements) by the appropriate constitutive law, then regularization is expected not to bias the measurement of the displacement field, but simply to dampen un-physical features of displacements (due e.g., to noise [22]). When the regularization length scale diverges, the displacement field converges to the actual displacement if the boundary conditions are appropriately determined. Hence regularization and identification meet in this limit.

The analysis of damage can be seen as the estimation of the local elastic properties of each volume element, for instance during unloading so that no plastic flow nor frictional sliding would occur [85,54]. Such a route can be followed, but it involves a very difficult inverse problem when the spatial resolution asked for the damage field is small. For such an approach, the number of unknowns tends to be very large [31 32 57.

A much more gratifying route is to search for damage as resulting from a constitutive law. As a simple example, damage $D(\boldsymbol{x})$ chosen to be a scalar field may be expressed as a function of an equivalent strain, $\epsilon_{e q}(\boldsymbol{x})$ (or the maximum over the loading history of this equivalent strain) to be computed from the local displacement field. In this case the unknowns are the parameters that will relate $D$ to $\epsilon_{e q}$, hence it is a 1D 
function that requires a few parameters to be well captured. For instance, in Refs. [126] 114 it was proposed to describe this function as a discrete sum of exponentials

$$
D\left(\epsilon_{e q}\right)=\sum_{i} a_{i}\left(1-\exp \left(-\epsilon_{e q} / \epsilon_{i}\right)\right)
$$

where $i=1, \ldots, m$ and the $\epsilon_{i}$ form a geometric series of characteristic strains. A common ratio of 2 gives a good scale separation, and $m$ is to be chosen so that it covers the range of equivalent strains encountered in a test. Asking for positive amplitudes $a_{i}$ summing up to 1 or less guarantees that damage remains thermodynamically acceptable.

The advantage of such a formulation is that it requires that all volume elements that experience the same equivalent strain are subjected to the same damage level. This condition is extremely strong and the subsequent reduction in the number of unknowns makes the problem much better posed than looking for arbitrary $D(\boldsymbol{x})$. Moreover, the accumulation of all available images acquired during a mechanical test (ideally containing numerous unloading phases) with the requirement that they all are accounted for by the same damage law adds up data but no other unknowns [114]13].

Once the problem has been formulated with a compact parametrization, different strategies can be considered for identifying the damage law [44, 31, 27]. Among those, the Finite Element Method Updating (FEMU) is very convenient and generic. To illustrate such an approach, the example of three-point flexure on plaster introduced in Section 3.2 and further analyzed in Sections 4.1 and 4.3 is again considered.

In the present DIC analysis a beam-like kinematics is implemented in a 2D-DIC code (e.g., with Euler Bernoulli beams 64 again) as would be used in finite element codes with beam elements. Each element of length $\ell$ has six degrees of freedom, namely, two translations and one rotation at both of its end sections. The kinematic basis $\boldsymbol{\mu}_{i}(\boldsymbol{x})$ (see Equation (2)) then corresponds to the shape functions associated with the previous degrees of freedom. Lagrange multipliers can be added when needed to enforce higher order continuities (e.g., curvature and derivative of curvature [65]) as discussed for the lumped damage approach. The measurement resolution is estimated by performing a DIC analysis between the reference image and a fictitious one altered by a synthetic noise of magnitude comparable to that observed in the images with a standard deviation of 5.5 gray levels. With the present DIC implementation, the standard displacement resolution is equal to $200 \mathrm{~nm}$ (or 0.003 pixel) when the element length $\ell$ varies from 25 to 75 pixels. The corresponding root mean square (RMS) correlation residual amounts precisely to the noise level (i.e., standard deviation of 5.5 gray levels).

Figure 12 shows the beam deflection, rotation and curvature fields as the applied displacement is increased in the 3-point flexural test of Section 3.2 In the present case, 40 beam elements of length $\ell=50$ pixels are considered. Displacements and rotations were chosen to be continuous. In order to allow for a degradation of the flexural stiffness under the contact point, the curvature was chosen to be discontinuous at the interface between the 18 th and 19 th element, and the 20th-21st element interface. For symmetry reasons, the central 19th-20th element interface was chosen to be continuous. The same DIC approach was also applied for smaller and larger elements (i.e., $\ell=25$ and 75 pixels). It is to be noted that this direct approach bears some similarities with the previous severity analysis. The major difference is that the left and right arms can exhibit a slight dissymmetry in the present case, whereas they were compelled to have an identical curvature in the severity analysis.

In the following FEMU is used to identify the parameters of a damage law, which assumes that the damage state is uniform per element. The modeling approach was 


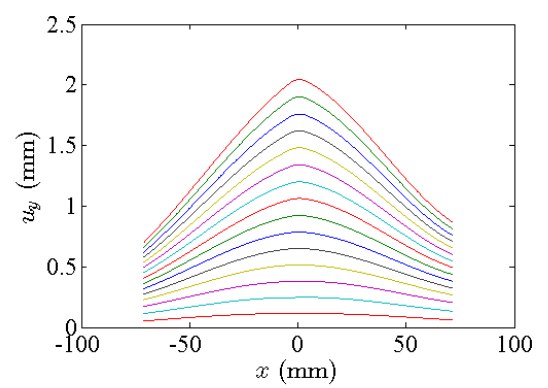

(a)

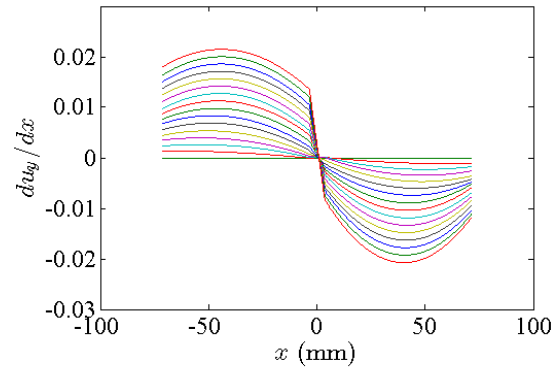

(b)

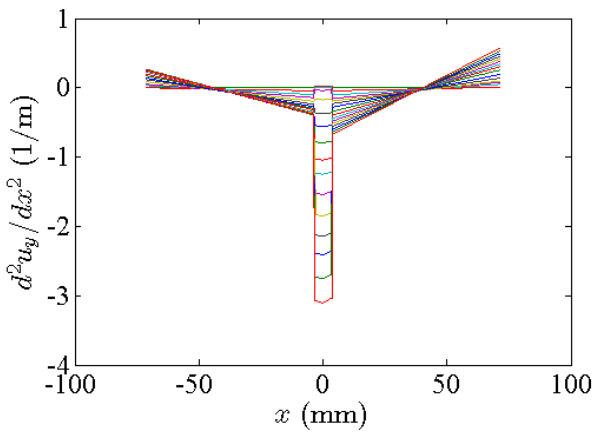

(c)

Fig. 12 Measured deflection (a), rotation (b) and curvature (c) fields with beam-DIC of the three-point flexural test on plasterboard, using beam elements of size $\ell=50$ pixels

set consistently with the DIC description, and hence only the two central elements of the beam are damaging, while the other elements remain undamaged. This would correspond to a nonlocal view point in which the element size plays the role of a characteristic length [65]. To initialize the identification procedure, it is possible to estimate the state of damage by resorting to the beam equation

$$
K_{f}(1-D) \frac{d^{2} u_{y}}{d x^{2}}=M
$$

where $K_{f}$ is the flexural rigidity, and $M$ the flexural moment. From DIC measurements (Figure 12, the mean curvature of the damaged elements $\kappa$ is determined. Since threepoint flexure is a statically determinate problem, the level of flexural moment in the damaged element is assessed by extrapolating the curvature profile for both damaged elements and evaluating the mean 'elastic' curvature $\kappa^{e}$, which is equal to $M / K_{f}$ so that the damage variable reads [65]

$$
D=1-\frac{\kappa^{e}}{\kappa}
$$


From this estimate, the damage level for each recorded picture is obtained as a function of the mean curvature in each damaged element (Figure 13). It is observed that the damage level is not identical in both elements, even though the curvature is very close. This is an indication of an asymmetric development of the crack, which is to be expected from the correlation residuals shown in Figure 6

The important step forward in the identification procedure is to assume that there is a unique relationship between damage and curvature. The damage growth law will be described by a three-parameter Weibull model 65

$$
D=1-\exp \left(-\left(\frac{\left\langle\kappa-\kappa_{t h}\right\rangle_{+}}{\kappa_{0}}\right)^{m}\right)
$$

where $\langle\bullet\rangle_{+}$denotes the positive part of $\bullet, \kappa_{t h}$ the threshold curvature below which no damage occurs, $\kappa_{0}$ a scale parameter, and $m$ the Weibull exponent. This phenomenological law is in rather good agreement with the experimental data (Figure 13) even though, as expected, it is not able to capture the effect of left-right asymmetry that is present in the test.

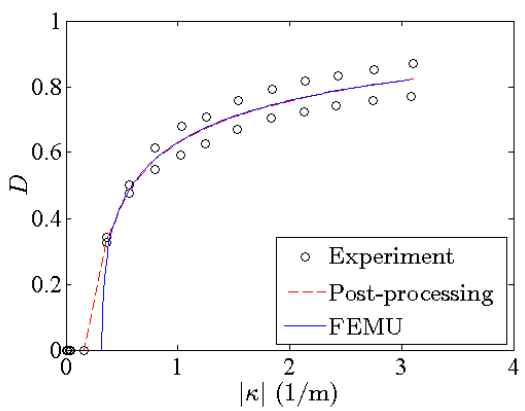

Fig. 13 Damage parameter as a function of the mean curvature per element of length $\ell=$ 50 pixels. The experimental data are the open circles, the dashed line corresponds to the direct fit by the three-parameter Weibull law. The solid line corresponds to the result of the identification via FEMU

FEMU consists of minimizing the chi-squared error with respect to the unknown parameters $\{\mathbf{p}\}$

$$
\chi^{2}(\{\mathbf{p}\})=\frac{1}{2}\left(\left\{\mathbf{u}^{m}\right\}-\left\{\mathbf{u}^{c}(\{\mathbf{p}\})\right\}\right)^{t}\left[\mathbf{C}_{u}\right]^{-1}\left(\left\{\mathbf{u}^{m}\right\}-\left\{\mathbf{u}^{c}(\{\mathbf{p}\})\right\}\right)
$$

where $\left\{\mathbf{u}^{m}\right\}$ is the column vector gathering all the measured degrees of freedom, $\left\{\mathbf{u}^{c}(\{\mathbf{p}\})\right\}$ the computed degrees of freedom for a given set of parameters $\{\mathbf{p}\}=$ $\left(\kappa_{t h}, \kappa_{0}, m\right)$, and $\left[\mathbf{C}_{u}\right]$ the covariance matrix associated with the measurement uncertainties. In the simulations, the measured degrees of freedom at both ends of the beam model are prescribed in addition to the deflection of the point load.

For the sake of simplicity, the covariance matrix is here assumed to be proportional to the identity matrix so that the previous minimization reduces to the standard nonlinear least squares error.

$$
\widehat{\chi}^{2}(\{\mathbf{p}\})=\frac{1}{2}\left(\left\{\mathbf{u}^{m}\right\}-\left\{\mathbf{u}^{c}(\{\mathbf{p}\})\right\}\right)^{t}\left(\left\{\mathbf{u}^{m}\right\}-\left\{\mathbf{u}^{c}(\{\mathbf{p}\})\right\}\right)
$$


The minimization is performed via a Gauss-Newton scheme by evaluating the sensitivity matrix gathering all sensitivity fields, which are computed from the incremental variation $\delta \mathbf{u}^{c}$ of the displacement field with respect to each parameter $p_{i}$

$$
\left[\mathbf{S}_{u}(\{\mathbf{p}\})\right]=\frac{\partial\left\{\mathbf{u}^{c}\right\}}{\partial\{\mathbf{p}\}}(\{\mathbf{p}\})
$$

so that for the current estimate of the sought parameters $\{\tilde{\mathbf{p}}\}$, their corrections $\delta\{\mathbf{p}\}$ are computed as

$$
\delta\{\mathbf{p}\}=\left[\mathbf{H}_{u}(\tilde{\mathbf{p}})\right]^{-1}\left[\mathbf{S}_{u}(\tilde{\mathbf{p}})\right]^{t}\left(\left\{\mathbf{u}^{m}\right\}-\left\{\mathbf{u}^{c}(\{\tilde{\mathbf{p}}\})\right\}\right)
$$

where $\left[\mathbf{H}_{u}(\tilde{\mathbf{p}})\right]$ is an estimate of the Hessian (i.e., $\left.\left[\mathbf{H}_{u}(\tilde{\mathbf{p}})\right]=\left[\mathbf{S}_{u}(\tilde{\mathbf{p}})\right]^{t}\left[\mathbf{S}_{u}(\tilde{\mathbf{p}})\right]\right)$. Figure 14 shows a comparison between the measured deflection and rotation with the fields of the FEMU procedure at convergence. A very good agreement is observed between the two series of fields. The root mean square error of the displacements is less than $7 \mu \mathrm{m}$ (i.e., 0.1 pixel). It is clearly higher than the measurement error (i.e., $200 \mathrm{~nm}$ ), thereby indicating that there exists a modeling error. One obvious origin (and certainly not the only one) is the dissymmetry observed between left and right arms. This is also confirmed by analyzing the RMS correlation residual of DIC analyses, which is equal to 9.9 gray levels on average over the whole load history, and reaches 14.6 gray levels when the crack is fully developed.

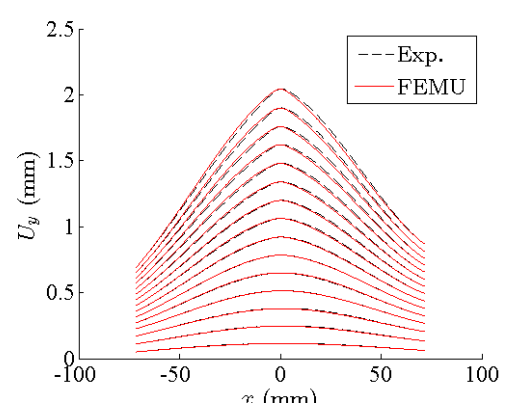

(a) Deflection

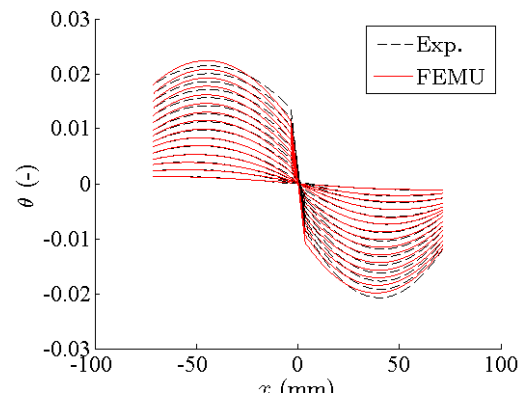

(b) Rotation

Fig. 14 Measured and identified fields via FEMU when $\ell=50$ pixels

When comparing the identified law from the post-processed measured curvature field or by FEMU (Figure 13, the high damage levels (i.e., $D>0.3$ ) predicted by the two identification routes coincide. Conversely, for damage levels close to the threshold curvature $\kappa_{t h}$, the two routes do not yield the same results. For the post-processed approach the threshold is very close to the largest level of the curvature that has not induced any damage. For FEMU, the threshold corresponds to the curvature level of first nonzero damage. The fact that cracking per se is very brittle leads to few data points for low levels of damage (i.e., $D<0.3$ ).

To assess the influence of the discretization, three different element sizes are investigated. Table 1 gathers the identification results when $\ell=25,50$ and 75 pixels. For these three sizes, the correlation residuals can hardly be distinguished from each 
other. The measured kinematics has an equivalent quality for the different discretizations. From the FEMU procedure, the optimal element length is $\ell=25$ pixels (i.e., $\approx 1.8 \mathrm{~mm}$ ) even though larger sizes lead to reasonable estimates, all of them leading to similar modeling errors when compared to the standard displacement resolutions, which is at least 30 times less than the identification error.

Table 1 Damage growth law parameters identified for different element sizes $\ell$, corresponding RMS correlation residuals and identification errors

\begin{tabular}{|c|c|ccc|c|}
\hline$\ell$ (pixels $/ \mathrm{mm})$ & $\sqrt{\left\langle\rho^{2}\right\rangle}(\mathrm{GL})$ & $\kappa_{t h}(1 / \mathrm{m})$ & $\kappa_{0}(1 / \mathrm{m})$ & $m$ & $\sqrt{2} \widehat{\chi}(\mu \mathrm{m})$ \\
\hline $25 / 1.8$ & 9.9 & 0.40 & 0.39 & 0.31 & 6.4 \\
\hline $50 / 3.6$ & 9.9 & 0.33 & 0.68 & 0.39 & 6.5 \\
\hline $75 / 5.3$ & 9.9 & 0.27 & 0.88 & 0.48 & 6.8 \\
\hline
\end{tabular}

When comparing the identified damage growth laws by both descriptions (see Figures 13 and 11(d)) the same order of magnitude is obtained for the damage parameter as a function of the considered curvature. It is worth remembering that in the case of lumped damage the curvature is evaluated for a single section (i.e., locally) as opposed to the present case in which it corresponds to the average of the damage element (i.e., nonlocally).

Last, let us note that the previous approach can be extended to a fully Integrated DIC technique or I-DIC [58,125]. This rewriting can be seen as a mere change of scales in which the identification problem is rewritten at the pixel/voxel (gray) level, and not at the nodal level of the measured displacement fields. It consists of solving the mechanical problem (and its displacement field $\boldsymbol{u}_{0}^{c}(\boldsymbol{x}, t)$ ) using a given set of parameters $\{\boldsymbol{p}\}$, and boundary conditions.

Two different routes can be followed. The first one consists of using closed-form solutions (e.g., in the case of cracks [125][128] or Brazilian tests [58]). Another more generic route is to use the sensitivity fields as the kinematic basis [129]100. The latter ones are introduced in Equation 22, namely, $\boldsymbol{\mu}_{i}(\boldsymbol{x}, t)=\partial \boldsymbol{u}^{c}(\boldsymbol{x}, t) / \partial p_{i}$. The unknown degrees of freedom then become the sought material parameters $\{\mathbf{u}\}=\{\mathbf{p}\}$. The DIC iteration is performed between the reference image $f(\boldsymbol{x})$ and the set of deformed images corrected by the displacement field $\boldsymbol{u}_{0}^{c}, \widetilde{g}(\boldsymbol{x}, t)=g\left(\boldsymbol{x}+\boldsymbol{u}_{0}^{c}(\boldsymbol{x}, t), t\right)$. The measured corrections $\delta p_{i}$ of these fields from DIC can be used to update the constitutive parameters according to

$$
p_{i} \leftarrow p_{i}+\delta p_{i}
$$

This defines one iteration of the I-DIC measurement. Iterations are carried out up to convergence for the set of material parameters $p_{i}$. This type of approach was used to tune the parameters of the growth law associated with an isotropic damage description [129]. It is worth noting that when weighted FEMU is considered (see Equation (17)), the two approaches are equivalent when the noise level remains small in comparison with the random texture of the studied material [100].

\subsection{Use of localization}

This section points toward a different use of regularization with a damage law. Damage was above defined as a representation of stiffness loss as being a (locally) uniform 
reduction in the elastic constants. This representation is one out of many equivalent ones (such as resulting from a set of microcracks). However, there are cases (e.g., softening) where the resulting constitutive law is prone to localization. That is to say, in spite of the fact that damage was assumed to be distributed over all material elements, at a large enough scale, a structure may be subjected to an instability where damage and strain localize onto a narrow region (e.g., band, crack). This phenomenon causes severe difficulties to finite-element modeling in the sense that the global response shows a mesh dependency, or in other words, it lacks objectivity [17]. This problem has given rise to a very abundant literature in order to propose remedies. The latter often involves (either implicitly or explicitly) an additional length scale below which the instability is suppressed [61].

The localization instability in fact can also be used to concentrate damage onto a very narrow support when coupled with DIC. If a crack is expected, it can be represented by a curve in $2 \mathrm{D}$ or a surface in $3 \mathrm{D}$ where damage is equal to unity. The support of the $D=1$ region has the property of being "sparse". Regularization with an elastic law or even with a damage law that does not exhibit softening will tend to spread out large strains. However, if the damage law gives rise to softening, strains tend to concentrate over narrow regions. In the field of applied mathematics and image analysis, the observation that a sparsity property may help filtering or restoring an image has been recognized rather recently, but has given rise to a very intense activity [36]. To promote sparsity, the key methodology is to use a cost function that is not convex. In fact, the condition for exhibiting a localization instability is precisely that the constitutive law used in the regularization gives rise to localization.

Such an approach has been implemented for the case of plasterboard subjected to three-point flexure. Here, one does not resort to a beam description, but rather to a 2D-DIC approach. The same region of interest and same mesh as used in Figure 4 is considered with a large regularization length (i.e., $\ell_{m}=50$ pixels). However, rather than using an elastic law (blurring the displacement or strain fields, see Figure 4 (a) and (c)), a damage law is used. Note however that the law itself is fictitious as is the estimate of strain that is artificially spread over elements. In the present case, for element sizes of 3 pixels, the damage law was chosen to be $D=D_{\infty}\left(1-\exp \left(-\left(\epsilon_{e q} / \epsilon_{c}\right)^{m}\right)\right)$, with $\epsilon_{e q}$ being the largest (positive) eigen strain, $\epsilon_{c}=0.06$, and $m=3$. The ultimate damage level $D_{\infty}$ is chosen to amount to 0.95 so that a residual stiffness exists even for large strains enabling the DIC problem to remain well-behaved. Figure 15 shows that such a law is able both to mark precisely the crack pattern (in spite of its complex shape) and simultaneously to smooth out spurious displacement fluctuations outside the cracked zones.

A similar procedure has been implemented in a regularized DVC code and applied to the three-point flexural experiment introduced in Section 3.1 (see Figure 2). The analyzed volume has a size of $336 \times 368 \times 336$ voxels or $8.4 \times 9.2 \times 8.4 \mathrm{~mm}^{3}$, with a regular C8 mesh of size $\ell=8$ voxels or $0.2 \mathrm{~mm}$ but with a larger regularization length (i.e., $\ell_{m}=60$ voxels) than that used in Section 4.1 This solution (with no damage) will serve as initialization to more refined calculations with $\ell_{m}=2 \ell_{b}=32$ voxels. The damage law has the following parameters $\epsilon_{c}=0.05, m=2$, and $D_{\infty}=0.9$. Note however that the displacement field is obtained through successive determinations where $\epsilon_{c}$ is reduced from 0.1 down to 0.05 and the resulting displacement field at one converged step is used as an initialization for the next step. Figure 16(a-b) shows the gray level residuals without the damage law (RMS level $=11.3$ gray levels) and with the damage law (RMS level $=10.5$ gray levels). There is a clear benefit with the damage law in terms of mean 


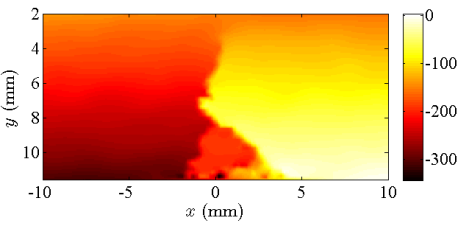

(a)

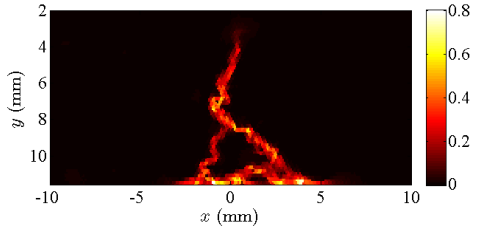

(b)

Fig. 15 (a) Horizontal displacement component $u_{x}$ (expressed in $\mu \mathrm{m}$ ) and (b) major principal strain $\epsilon_{e q}$ maps as obtained from DIC regularized with a localizing damage law and a large regularization length $\ell_{m}=\ell_{b}=50$ pixels

correlation residual and localization of the complex cracked surfaces. When compared with the thresholded deformed configuration (Figure 16(c)), it is advantageous to use DVC analyses not only to reveal more clearly the crack but also to get a quantitative estimate of the openings via the equivalent strain field shown in Figure 16(d-e). With the damage law, the corresponding field is more localized, and some features can be observed (i.e., debonding between plaster and paper lining on the bottom part of the sample and the second 'foot' of the $\lambda$-shaped crack), which cannot be seen when the damage law is not used. The present study confirms the observations that were obtained in the previous 2D analysis (Figure 15), and they show that in this particular case what can be quantified on the external surface is essentially identical through the whole thickness of the sample.

The 1D equivalent of such approaches would reduce precisely to a lumped damage approach. So no new observation is to be added here but it is worth emphasizing that the DIC usage of damage in order to enhance localization may meet that formalism chosen for its operational representation in numerical models. The convergence of objectives and procedures is striking.

\section{Summary and Outlook}

Among the different measurement routes that were introduced herein to characterize damage, two of them were used. First, detection issues were illustrated to observe and quantify the development of physical damage (e.g., microcracks and microvoids) by using 2D and 3D images, which are subsequently processed. More emphasis was put on microcracks rather than microvoids since they are generally more challenging in terms of detectability and quantification. Second, the coupling between elasticity and damage, which is one of the ways to assess damage fields, was used to identify the parameters of damage growth laws. The choice of either approach is usually dictated by the modeling framework, which relies on the scale of observation and the inclination of the scientist. The identification of the (mechanical) damage law was mainly illustrated in the simple case of beam geometries for the sake of simplicity. It can be noted that such approaches have also been applied to other materials and experimental configurations (e.g., ductile metals and alloys [86 3 |90 147, composite materials [3 57 147], cement and concrete [15 3 [44 147]). Last, the issue of properly handling localized phenomena, 


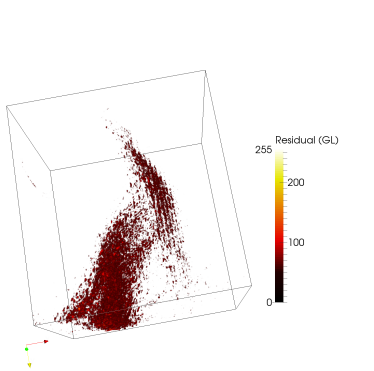

(a)

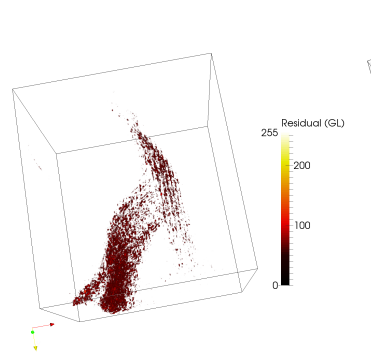

(b)

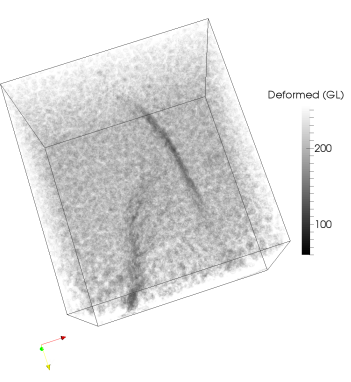

(c)

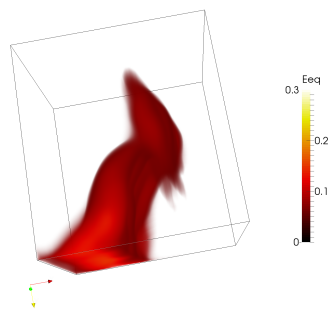

(d)

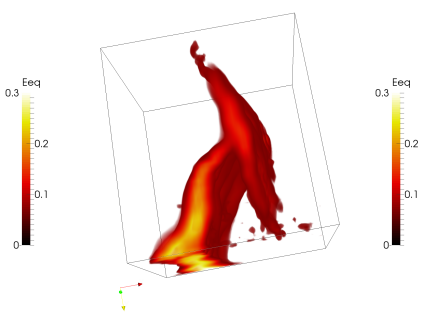

(e)

Fig. 16 3D renderings of gray level correlation residuals without (a) and with (b) a damage law. (c) Thresholded cracked configuration in which the crack appears very faintly. Major principal strain $\epsilon_{e q}$ without (d) and with (e) a damage law. All the results have been obtained via DVC regularized with a large regularization length $\ell_{m}=\ell_{b}=60$ voxels

so that the chosen discretization remains compatible with the energy balance has been discussed, for instance, for lumped damage mechanics (for beams).

To perform all the analyses reported in the present paper, only one full-field measurement technique (i.e., DIC and DVC) was used. Further, the identification procedures were tailored to the specific features associated with physical and mechanical damage. It is worth noting that there exist other full-field measurement and identification procedures that could have been used [46]. The key choice was to link as strongly as possible both steps, namely, measurement and identification. Global approaches to DIC and DVC are one way to achieve this goal so that seamless procedures can be implemented to bridge the gap between experiments and numerical simulations.

In terms of damage models, only very simple ones were used herein for illustration purposes, but also because complexity has to be dealt with special care. Mostly 2D displacement fields were used in the examples. However, the proposed identification procedures are generic; they can (and will) be generalized to 3D surface and volume measurements. As discussed in the introduction, different scales can be considered at the measurement and modeling step. For damage detection, the scale will determine the spatial resolution of the sought mechanism. For the damage models, they were essentially written at the level of the volume element of continuum mechanics. Other choices could have been made. Similarly, most of the presented experiments have been conducted on plasterboard samples. It should not be concluded that the procedures discussed herein can only be applied to this type of material. They are indeed generic 
and can be tailored to the underlying damage mechanisms at play and to the chosen modeling strategy (see, e.g., Refs. [62]). They assume different expressions for slender geometries (e.g., beams, plates) and for bulk solids but the underlying philosophy remains the same.

All these developments are geared toward the emergence of simulation-based engineering sciences. Among the various challenges [109, one of them is associated with multiscale and multi-physics models. Continuum damage mechanics is one area of solid mechanics that needs further developments to reach a level of confidence sufficiently high for engineers to use the models to design (damage-tolerant) structures. Real-time integration of simulation methods with measurement systems is another issue to be addressed. To achieve this goal, robust model identification and validation procedures need to be improved and made robust.

Acknowledgements This work has been financially supported by Saint-Gobain Recherche, by ANRT through grant no. 2010/567, and by the French "Agence Nationale de la Recherche," through the "Investissements d'avenir" program (ANR-10-EQPX-37 MATMECA grant). The authors would like to warmly thank Drs. Jean-Louis Chaboche, André Dragon, and Prof. Jean Lemaitre for numerous discussions on issues introduced in the present paper.

\section{References}

1. Abanto-Bueno, J., Lambros, J.: Experimental determination of cohesive failure properties of a photodegradable copolymer. Exp. Mech. 45(2), 144-152 (2005)

2. Abry, J., Bochard, S., Chateauminois, A., Salvia, M., Giraud, G.: In situ detection of damage in CFRP laminates by electrical resistance measurements. Compos. Sci. Tech. 59, 925-935 (1999)

3. Allix, O., Hild, F. (eds.): Continuum Damage Mechanics of Materials and Structures. Elsevier, Amsterdam (the Netherlands) (2002)

4. Ambrose, J., Hounsfield, G.: Computerized transverse axial tomography. Br. J. Radiol. 46(542), 148-149 (1973)

5. Ashby, M.: Physical modeling of materials problems. Mater. Sci. Tech. 8, 102-111 (1992)

6. Ashby, M., Dyson, B.: Creep damage mechanics and micromechanisms. Tech. Rep. 77, Natl. Phys. Lab. UK (1984)

7. Avril, S., Bonnet, M., Bretelle, A., Grédiac, M., Hild, F., Ienny, P., Latourte, F., Lemosse, D., Pagano, S., Pagnacco, E., Pierron, F.: Overview of identification methods of mechanical parameters based on full-field measurements. Exp. Mech. 48(4), 381-402 (2008)

8. Babout, L., Maire, E., Buffière, J., Fougères, R.: Characterisation by X-ray computed tomography of decohesion, porosity growth and coalescence in model metal matrix composites. Acta Mater. 49(11), 2055-2063 (2001)

9. Babout, L., Maire, E., Fougères, R.: Damage initiation in model metallic materials: X-ray tomography and modelling. Acta Mater. 52, 2475-2487 (2004)

10. Baruchel, J., Buffière, J., Maire, E., Merle, P., Peix, G. (eds.): X-Ray Tomography in Material Sciences. Hermes Science, Paris (France) (2000)

11. Bay, B., Smith, T., Fyhrie, D., Saad, M.: Digital volume correlation: three-dimensional strain mapping using X-ray tomography. Exp. Mech. 39, 217-226 (1999)

12. Beevers, C.: Advances in Crack Length Measurement. EMAS, West Midlands (UK) (1982)

13. Ben Azzouna, M., Périé, J., Guimard, J., Hild, F., Roux, S.: On the identification and validation of an anisotropic damage model by using full-field measurements. Int. J. Damage Mech. 20(8), 1130-1150 (2011)

14. Bentz, D., Martys, N., Stutzman, P., Levenson, M., Garboczi, E., Dunsmuir, J., Schwartz, L.: X-ray microtomography of an ASTM C109 mortar exposed to sulfate attack. In: S. Diamond, S. Mindess, F. Glasser, L. Roberts (eds.) MRS Symposium, vol. 370, pp. 77-82. MRS, Pittsburg (PA), USA (1995)

15. Berthaud, Y.: Damage measurements in concrete via an ultrasonic technique. Part I experiment. Cement Concr. Res. 21(1), 73-82 (1991) 
16. Besnard, G., Hild, F., Roux, S.: "Finite-element" displacement fields analysis from digital images: Application to Portevin-Le Châtelier bands. Exp. Mech. 46, 789-803 (2006)

17. Billardon, R., Doghri, I.: Prediction of macro-crack initiation by damage localization. C. R. Acad. Sci. Paris, II 308(4), 347-352 (1989)

18. Bontaz-Carion, J., Pellegrini, Y.: X-ray microtomography analysis of dynamic damage in tantalum. Adv. Eng. Mat. 8(6), 480-486 (2006)

19. Borbély, A., Dzieciol, K., Sket, F., Isaac, A., di Michiel, M., Buslaps, T., Kaysser-Pyzalla, A.: Characterization of creep and creep damage by in-situ microtomography. JOM 63(7), 78-84 (2011)

20. Bornert, M., Bretheau, T., Gilormini, P. (eds.): Homogenization in Mechanics of Materials. Lavoisier, Paris (France) (2008)

21. Borré, G., Maier, G.: On linear versus nonlinear flaw rules in strain localization analysis. Meccanica 24, 36-41 (1989)

22. Bouterf, A., Roux, S., Hild, F., Adrien, J., Maire, E.: Digital volume correlation applied to X-ray tomography images from spherical indentation tests on lightweight gypsum. Strain 50(5), 444-453 (2014)

23. Budiansky, B., O'Connell, R.: Elastic moduli of a cracked system. Int. J. Solids Struct. 12, 81-97 (1976)

24. Buffière, J., Maire, E., Adrien, J., Masse, J., Boller, E.: In situ experiments with X ray tomography: an attractive tool for experimental mechanics. Exp. Mech. 50(3), 289-305 (2010)

25. Buffière, J., Maire, E., Cloetens, P., Lormand, G., Fougères, R.: Characterisation of internal damage in a MMCp using X-ray synchrotron phase contrast microtomography. Acta Mater. 47(5), 1613-1625 (1999)

26. Burr, A., Hild, F., Leckie, F.: Micro-mechanics and continuum damage mechanics. Arch. Appl. Mech. 65(7), 437-456 (1995)

27. Chalal, H., Meraghni, F., Pierron, F., Grédiac, M.: Direct identification of the damage behaviour of composite materials using the virtual fields method. Composites: Part A 35, 841-848 (2004)

28. Chermant, J., Boitier, G., Darzens, S., Coster, M., Chermant, L.: Damage morphological parameters. Image Anal. Stereol. 20, 207-211 (2001)

29. Chu, T., Ranson, W., Sutton, M., Peters, W.: Applications of digital-image-correlation techniques to experimental mechanics. Exp. Mech. 3(25), 232-244 (1985)

30. Cipollina, A., López-Inojosa, A., Flórez-López, J.: A simplified damage mechanics approach to nonlinear analysis of frames. Comput. Struct. 54(6), 1113-1126 (1995)

31. Claire, D., Hild, F., Roux, S.: Identification of damage fields using kinematic measurements. C. R. Mécanique 330, 729-734 (2002)

32. Claire, D., Hild, F., Roux, S.: A finite element formulation to identify damage fields: The equilibrium gap method. Int. J. Num. Meth. Engng. 61(2), 189-208 (2004)

33. Claire, D., Hild, F., Roux, S.: Identification of a damage law by using full-field displacement measurements. Int. J. Damage Mech. 16(2), 179-197 (2007)

34. Cocks, A., Leckie, F.: Creep Constitutive Equations for Damaged Materials, vol. 25, pp. 239-294. Academic Press, New York (USA) (1987)

35. Coster, M., Chermant, J.L.: Image analysis and mathematical morphology for civil engineering materials. Cement Concr. Compos. 23(2-3), 133-151 (2001)

36. Daubechies, I., Defrise, M., De Mol, C.: An iterative thresholding algorithm for linear inverse problems with a sparsity constraint. Comm. Pure Appl. Math. 57(11), 1413-1457 (2004)

37. Denoual, C., Hild, F.: A damage model for the dynamic fragmentation of brittle solids. Comp. Meth. Appl. Mech. Eng. 183, 247-258 (2000)

38. Desrues, J., Viggiani, G., Bésuelle, P. (eds.): Advances in X-ray Tomography for Geomaterials. Wiley / ISTE (2006)

39. Doghri, I., Billardon, R.: Investigation of localization due to damage in elasto-plastic materials. Mech. Mater. 19, 129-149 (1995)

40. Eshelby, J.: The elastic field outside an ellipsoidal inclusion. Proc. Roy. Soc. London A 252, 561-569 (1959)

41. Evans, A.: Perspectives on the development of high-toughness ceramics. J. Am. Ceram. Soc. 73(2), 187-206 (1990)

42. Fedele, R., Raka, B., Hild, F., Roux, S.: Identification of adhesive properties in glare assemblies by digital image correlation. J. Mech. Phys. Solids 57, 1003-1016 (2009) 
43. Flannery, B., Deckman, H., Roberge, W., D'Amico, K.: Three-dimensional microtomography. Science 237, 1439-1444 (1987)

44. Geers, M., De Borst, R., Peijs, T.: Mixed numerical-experimental identification of nonlocal characteristics of random-fibre-reinforced composites. Compos. Sci. Tech. 59, 1569$1578(1999)$

45. Gras, R., Leclerc, H., Hild, F., Roux, S., Schneider, J.: Identification of a set of macroscopic elastic parameters in a $3 \mathrm{D}$ woven composite: Uncertainty analysis and regularization. Int. J. Solids Struct. 55, 2-16 (2015)

46. Grédiac, M., Hild, F. (eds.): Full-Field Measurements and Identification in Solid Mechanics. ISTE / Wiley, London (UK) (2012)

47. Hall, F., Hayhurst, D.: Continuum damage mechanics modeling of high temperature deformation and failure in a pipe weldment. Proc. Roy. Soc. London A 433, 383-403 (1991)

48. Helfen, L., Baumbach, T., Mikulík, P., Kiel, D., Pernot, P., Cloetens, P., Baruchel, J.: High-resolution three-dimensional imaging of flat objects by synchrotron-radiation computed laminography. Appl. Phys. Lett. 86(7), 071,915 (2005)

49. Helfen, L., Morgeneyer, T., Xu, F., Mavrogordato, M., Sinclair, I., Schillinger, B., Baumbach, T.: Synchrotron and neutron laminography for three-dimensional imaging of devices and flat material specimens. Int. J. Mat. Res. 2012(2), 170-173 (2012)

50. Helfen, L., Myagotin, A., Mikulík, P., Pernot, P., Voropaev, A., Elyyan, M., Di Michiel, M., Baruchel, J., Baumbach, T.: On the implementation of computed laminography using synchrotron radiation. Rev. Sci. Instrum. 82(063702) (2011)

51. Helfen, L., Myagotin, A., Rack, A., Pernot, P., Mikulík, P., Di Michiel, M., Baumbach, T.: Synchrotron-radiation computed laminography for high-resolution three-dimensional imaging of flat devices. Phys. Stat. Sol. (a) 204, 2760-2765 (2007)

52. Hellier, C.: Handbook of Nondestructive Evaluation. McGraw Hill, New York, NY (USA) (2001)

53. Hild, F., Burr, A., Leckie, F.: Fiber breakage and fiber pull-out of fiber-reinforced ceramicmatrix composites. Eur. J. Mech. A/Solids 13(6), 731-749 (1994)

54. Hild, F., Burr, A., Leckie, F.: Matrix cracking and debonding in ceramic-matrix composites. Int. J. Solids Struct. 33(8), 1209-1220 (1996)

55. Hild, F., Fanget, A., Adrien, J., Maire, E., Roux, S.: Three dimensional analysis of a tensile test on a propellant with digital volume correlation. Arch. Mech. 63(5-6), 1-20 (2011)

56. Hild, F., Larsson, P., Leckie, F.: Localization due to damage in fiber reinforced composites. Int. J. Solids Struct. 29(24), 3221-3238 (1992)

57. Hild, F., Périé, J.N., Roux, S.: Evaluating damage with digital image correlation: C. Applications to composite materials. In: G. Voyiadjis (ed.) Handbook of Damage Mechanics, pp. 1301-1322. Springer, New York, NY (USA) (2014)

58. Hild, F., Roux, S.: Digital image correlation: From measurement to identification of elastic properties - A review. Strain 42, 69-80 (2006)

59. Hild, F., Roux, S.: Comparison of local and global approaches to digital image correlation. Exp. Mech. 52(9), 1503-1519 (2012)

60. Hild, F., Roux, S.: Digital image correlation. In: P. Rastogi, E. Hack (eds.) Optical Methods for Solid Mechanics. A Full-Field Approach, pp. 183-228. Wiley-VCH, Weinheim (Germany) (2012)

61. Hild, F., Roux, S.: Evaluating damage with digital image correlation: A. Introductory remarks and detection of physical damage. In: G. Voyiadjis (ed.) Handbook of Damage Mechanics, pp. 1255-1275. Springer, New York, NY (USA) (2014)

62. Hild, F., Roux, S.: Evaluating damage with digital image correlation: B. From physical to mechanical damage. In: G. Voyiadjis (ed.) Handbook of Damage Mechanics, pp. 12771299. Springer, New York, NY (USA) (2014)

63. Hild, F., Roux, S., Bernard, D., Hauss, G., Rebai, M.: On the use of 3D images and 3D displacement measurements for the analysis of damage mechanisms in concrete-like materials. In: J. Van Mier, G. Ruiz, C. Andrade, R. Yu, X. Zhang (eds.) FraMCoS-8, pp. $1-11(2013)$

64. Hild, F., Roux, S., Gras, R., Guerrero, N., Marante, M., Flórez-López, J.: Displacement measurement technique for beam kinematics. Opt. Lasers Eng. 47, 495-503 (2009)

65. Hild, F., Roux, S., Guerrero, N., Marante, M., Florez-Lopez, J.: Calibration of constitutive models of steel beams subject to local buckling by using digital image correlation. Eur. J. Mech. A/Solids 30, 1-10 (2011) 
66. Hounsfield, G.: Computerized transverse axial scanning (tomography). 1. Description of system. Br. J. Radiol. 46(552), 1016-22 (1973)

67. Huppmann, M., Camin, B., Pyzalla, A.R., Reimers, W.: In-situ observation of creep damage evolution in $\mathrm{Al}-\mathrm{Al}_{2} \mathrm{O}_{3} \mathrm{MMCs}$ by synchrotron X-ray microtomography. Int. J. Mat. Res. 101, 372-379 (2010)

68. Isaac, A., Sket, F., Reimers, W., Camin, B., Sauthoff, G., Pyzalla, A.: In situ 3D quantification of the evolution of creep cavity size, shape, and spatial orientation using synchrotron X-ray tomography. Mat. Sci. Eng. A478(1-2), 108-118 (2008)

69. Kachanov, L.: Time of the rupture process under creep conditions. Bull. SSR Acad. Sci., Division of Technical Sciences (in Russian) 8, 26-31 (1958)

70. Kachanov, L.: Rupture time under creep conditions. In: J. Radok (ed.) Problems of Continuum Mechanics, pp. 202-218. SiAM, Philadelphia (USA) (1961)

71. Kachanov, L.: Crack and damage growth in creep - a combined approach. Int. J. Fract. 16(4), R179-R181 (1980)

72. Kak, A., Slaney, M.: Principles of Computerized Tomographic Imaging. Society of Industrial and Applied Mathematics (2001)

73. Krajcinovic, D.: Damage Mechanics. North Holland Series in Appl. Math. and Mech. Elsevier, Amsterdam (the Netherlands) (1996)

74. Landis, E., Zhang, T., Nagy, E., Nagy, G., Franklin, W.R.: Cracking, damage and fracture in four dimensions. Mat. Struct. 40, 357-364 (2007)

75. Landron, C., Bouaziz, O., Maire, E., Adrien, J.: Characterization and modeling of void nucleation by interface decohesion in dual phase steels. Scripta Mat. 63, 973-976 (2010)

76. Landron, C., Maire, E., Adrien, J., Bouaziz, O., Di Michiel, M., Cloetens, P., Suhonen, H.: Resolution effect on the study of ductile damage using synchrotron X-ray tomography. Nuclear Instruments \& Methods in Physics Research Section B-Beam Interactions with Materials and Atoms 284, 15-18 (2012)

77. Landron, C., Maire, E., Bouaziz, O., Adrien, J., Lecarme, L., Bareggi, A.: Validation of void growth models using X-ray microtomography characterization of damage in dual phase steels. Acta Mat. 59(20), 7564-7573 (2011)

78. Leclerc, H., Périé, J., Hild, F., Roux, S.: Digital volume correlation: What are the limits to the spatial resolution? Mech. \& Indust. 13, 361-371 (2012)

79. Leclerc, H., Périé, J., Roux, S., Hild, F.: Integrated digital image correlation for the identification of mechanical properties. In: A. Gagalowicz, W. Philips (eds.) MIRAGE 2009, vol. LNCS 5496, pp. 161-171. Springer, Berlin (2009)

80. Leclerc, H., Périé, J., Roux, S., Hild, F.: Voxel-scale digital volume correlation. Exp. Mech. 51(4), 479-490 (2011)

81. Lemaitre, J.: Théorie mécanique de l'endommagement isotrope appliqué à la fatigue des métaux. In: B. Halphen, Q. Nguyen (eds.) Matériaux et structures sous chargement cyclique, pp. 133-144. Association Amicale des Ingénieurs Anciens Elèves de l'E.N.P.C. (1978)

82. Lemaitre, J.: A Course on Damage Mechanics. Springer-Verlag, Berlin (Germany) (1992)

83. Lemaitre, J., Cordebois, J., Dufailly, J.: Elasticity and damage coupling. C. R. Acad Sci. Paris, Series B 288(23), 391-394 (1979)

84. Lemaitre, J., Desmorat, R.: Engineering Damage Mechanics. Springer, Berlin (Germany) (2005)

85. Lemaitre, J., Dufailly, J.: Modélisation et identification de l'endommagement plastique des métaux. In: 3e congrès français de mécanique. Grenoble (France) (1977)

86. Lemaitre, J., Dufailly, J.: Damage measurements. Eng. Fract. Mech. 28(5-6), 643-661 (1987)

87. Lemaitre, J., Marquis, D.: Modeling complex behavior of metals by the 'state-kinetic coupling theory'. ASME J. Eng. Mater. Tech. 114, 250-254 (1992)

88. Limodin, N., Réthoré, J., Buffière, J., Gravouil, A., Hild, F., Roux, S.: Crack closure and stress intensity factor measurements in nodular graphite cast iron using $3 \mathrm{D}$ correlation of laboratory X ray microtomography images. Acta Mat. 57(14), 4090-4101 (2009)

89. Locker, J., Prenter, P.: Regularization with differential operators. I. General theory. J. Math. Anal. Appl. 74, 504-529 (1980)

90. Maire, E.: Quantitative measurement of damage, pp. 79-108. Presses Ecole des Mines de Paris, Paris (France) (2003)

91. Maire, E., Bouaziz, O., Di Michiel, M., Verdu, C.: Initiation and growth of damage in a dual-phase steel observed by X-ray microtomography. Acta Mat. 56(18), 4954-4964 (2008) 
92. Maire, E., Carmona, V., Courbon, J., Ludwig, W.: Fast X-ray tomography and acoustic emission study of damage in metals during continuous tensile tests. Acta Mater. 55, 6806-6815 (2007)

93. Maire, E., Morgeneyer, T., Landron, C., Adrien, J., Helfen, L.: Bulk evaluation of ductile damage development using high resolution tomography and laminography. C. R. Physique 13, 328-336 (2012)

94. Maire, E., Withers, P.J.: Quantitative X-ray tomography. Int. Mat. Rev. 59(1), 1-43 (2014)

95. Marante, M., Florez-Lopez, J.: Model of damage for RC elements subjected to biaxial bending. Eng. Struct. 24(9), 1141-1152 (2002)

96. Marante, M., Flórez-López, J.: Three-dimensional analysis of reinforced concrete frames based on lumped damage mechanics. Int. J. Solids Struct. 40(19), 5109-5023 (2003)

97. Marigo, J., Pham, K., Sicsic, P.: Gradient damage models: a relevant approach to account for nucleation and propagation of cracks. Int. J. Fract. (2015)

98. Martin, C., Josserond, C., Salvo, L., Blandin, J., Cloetens, P., Boller, E.: Characterisation by X-ray micro-tomography of cavity coalescence during superplastic deformation. Scripta Mat. 42(4), 375-381 (2000)

99. Mathieu, F., Aimedieu, P., Guimard, J., Hild, F.: Identication of interlaminar fracture properties of a composite laminate using local full-field kinematic measurements and finite element simulations. Comp. Part A 49, 203-213 (2013)

100. Mathieu, F., Leclerc, H., Hild, F., Roux, S.: Estimation of elastoplastic parameters via weighted FEMU and integrated-DIC. Exp. Mech. (DOI:10.1007/s11340-014-98889) (2015)

101. McNeill, S., Peters, W., Sutton, M.: Estimation of stress intensity factor by digital image correlation. Eng. Fract. Mech. 28(1), 101-112 (1987)

102. Mills, K.: Fractography, vol. 12. ASM International, Materials Park, OH (USA) (1991)

103. Moffat, A., Wright, P., Helfen, L., Baumbach, T., Johnson, G., Spearing, S., Sinclair, I.: In situ synchrotron computed laminography of damage in carbon fibre-epoxy [90/0]s laminates. Scripta Mat. 62, 97-100 (2010)

104. Morgeneyer, T., Besson, J.: Flat to slant ductile fracture transition: tomography examination and simulations using shear controlled void nucleation. Scripta Mat. 65, 1002-1005 (2011)

105. Morgeneyer, T., Helfen, L., Mubarak, H., Hild, F.: 3D digital volume correlation of synchrotron radiation laminography images of ductile crack initiation: An initial feasibility study. Exp. Mech. 53(4), 543-556 (2013)

106. Morgeneyer, T., Helfen, L., Sinclair, I., Proudhon, H., Xu, F., Baumbach, T.: Ductile crack initiation and propagation assessed via in situ synchrotron radiation computed laminography. Scripta Mat. 65, 1010-1013 (2011)

107. Morgeneyer, T., Taillandier-Thomas, T., Helfen, L., Baumbach, T., Sinclair, I., Roux, S., Hild, F.: In situ 3D observation of early strain localisation during failure of thin Al alloy (2198) sheet. Acta Mat. 69, 78-91 (2014)

108. Odqvist, F.: On the theories of creep rupture. In: Int. Symp. on Second Order Elasticity, Plasticity and Fluid Dynamics. Pergamon Press, London (UK) (1964)

109. Panel, B.R.: Simulation-based engineering sciences. Final report, NFS (www.nsf.gov/pubs/reports/sbes final report.pdf) (2006)

110. Peerlings, R., de Borst, R., Brekelmans, W., de Vree, J.: Gradient-enhanced damage for quasi-brittle materials. Int. J. Num. Meth. Eng. 39, 3391-3403 (1996)

111. Peters, W., Ranson, W.: Digital imaging techniques in experimental stress analysis. Opt. Eng. 21, 427-431 (1982)

112. Pijaudier-Cabot, G., Bažant, Z.: Nonlocal damage theory. ASCE J. Eng. Mech. 113(10), 1512-1533 (1987)

113. Prabhakaran, R.: Damage assessment through electrical resistance measurement in graphite fiber-reinforced composites. Exp. Tech. 14(1), 16-20 (1990)

114. Périé, J., Leclerc, H., Roux, S., Hild, F.: Digital image correlation and biaxial test on composite material for anisotropic damage law identification. Int. J. Solids Struct. 46, 2388-2396 (2009)

115. Puncreobutr, C., Lee, P.D., Kaye, M., Balint, D., Farrugia, D., Connolley, T., Lin, J.: Quantifying damage accumulation during the hot deformation of free-cutting steels using ultra-fast synchrotron tomography, vol. 33, p. 012038 (2012)

116. Pyzalla, A., Camin, B., Buslaps, T., Di Michiel, M., Kaminski, H., Kottar, A., Pernack, A., Reimers, W.: Simultaneous tomography and diffraction analysis of creep damage. Science 308(5718), 92-95 (2005) 
117. Rabotnov, Y.: On the Equations of State for Creep, pp. 307-315. McMillan, New York (USA) (1963)

118. Rannou, J., Limodin, N., Réthoré, J., Gravouil, A., Ludwig, W., Baïetto, M., Buffière, J., Combescure, A., Hild, F., Roux, S.: Three dimensional experimental and numerical multiscale analysis of a fatigue crack. Comp. Meth. Appl. Mech. Eng. 199, 1307-1325 (2010)

119. Rastogi, P. (ed.): Photomechanics, Topics in Applied Physics, vol. 77. Springer, Berlin (Germany) (2000)

120. Rastogi, P., Hack, E. (eds.): Optical Methods for Solid Mechanics. A Full-Field Approach. Wiley-VCH, Berlin (2012)

121. Rice, J.: The Localization of Plastic Deformations, pp. 207-220. North-Holland (1976)

122. Rice, J., Rudnicki, J.: A note on some features of the theory of localization of deformation. Int. J. Solids Struct. 16, 597-605 (1980)

123. Rougelot, T., Burlion, N., Bernard, D., Skoczylas, F.: About microcracking due to leaching in cementitious composites: X-ray microtomography and numerical approach. Cement Concr. Comp. 40, 271-283 (2010)

124. Roux, S., Hild, F.: On the relevance of mean field to continuum damage mechanics. Int. J. Fract. 116(3), 219-229 (2002)

125. Roux, S., Hild, F.: Stress intensity factor measurements from digital image correlation: post-processing and integrated approaches. Int. J. Fract. 140(1-4), 141-157 (2006)

126. Roux, S., Hild, F.: Digital image mechanical identification (DIMI). Exp. Mech. 48(4), 495-508 (2008)

127. Roux, S., Hild, F., Viot, P., Bernard, D.: Three dimensional image correlation from X-ray computed tomography of solid foam. Comp. Part A 39(8), 1253-1265 (2008)

128. Roux, S., Réthoré, J., Hild, F.: Digital image correlation and fracture: An advanced technique for estimating stress intensity factors of $2 \mathrm{D}$ and $3 \mathrm{D}$ cracks. J. Phys. D: Appl. Phys. 42, 214,004 (2009)

129. Réthoré, J.: A fully integrated noise robust strategy for the identification of constitutive laws from digital images. Int. J. Num. Meth. Eng. 84(6), 631-660 (2010)

130. Réthoré, J., Estevez, R.: Identification of a cohesive zone model from digital images at the micron-scale. J. Mech. Phys. Solids 61(6), 1407-1420 (2013)

131. Réthoré, J., Roux, S., Hild, F.: An extended and integrated digital image correlation technique applied to the analysis fractured samples. Eur. J. Comput. Mech. 18, 285-306 (2009)

132. Rupil, J., Roux, S., Hild, F., Vincent, L.: Fatigue microcrack detection with digital image correlation. J. Strain Analysis 46(6), 492-509 (2011)

133. Salvo, L., Cloetens, P., Maire, E., Zabler, S., Blandin, J.J., Buffiere, J.Y., Ludwig, W., Boller, E., Bellet, D., Josserond, C.: X-ray micro-tomography an attractive characterisation technique in materials science. Nucl. Inst. Meth. Phys. Res. B 200, 273-286 (2003)

134. Sanchez-Palentia, E., Zaoui, A.: Homogenization Techniques for Composite Media. Lecture Notes in Physics 272. Springer-Verlag, Berlin (1987)

135. Shen, Y., Morgeneyer, T., Garnier, J., Allais, L., Helfen, L., Crépin, J.: Three-dimensional quantitative in situ study of crack initiation and propagation in AA6061 aluminum alloy sheets via synchrotron laminography and finite-element simulations. Acta Mat. 61(7), 2571-2582 (2013)

136. Smith, T., Bay, B., Rashid, M.: Digital volume correlation including rotational degrees of freedom during minimization. Exp. Mech. 42(3), 272-278 (2002)

137. Stock, S.: Recent advances in X-ray microtomography applied to materials. Int. Mat. Rev. 53(3), 129-181 (2008)

138. Stock, S.R., Naik, N.K., Wilkinson, A.P., Kurtis, K.E.: X-ray microtomography (microCT) of the progression of sulfate attack of cement paste. Cement Concr. Res. 32(10), $1673-1675$ (2002)

139. Sutton, M.: Computer vision-based, noncontacting deformation measurements in mechanics: A generational transformation. Appl. Mech. Rev. 65(AMR-13-1009, 050802) (2013)

140. Sutton, M., Orteu, J., Schreier, H.: Image correlation for shape, motion and deformation measurements: Basic Concepts, Theory and Applications. Springer, New York, NY (USA) (2009)

141. Sutton, M., Wolters, W., Peters, W., Ranson, W., McNeill, S.: Determination of displacements using an improved digital correlation method. Im. Vis. Comp. 1(3), 133-139 (1983) 
142. Sutton, M.A., Yan, J., Deng, X., Cheng, C.S., Zavattieri, P.: Three-dimensional digital image correlation to quantify deformation and crack-opening displacement in ductile aluminum under mixed-mode I/III loading. Opt. Eng. 46(5), 051,003-051,003-17 (2007)

143. Taillandier-Thomas, T., Roux, S., Morgeneyer, T., Hild, F.: Localized strain field measurement on laminography data with mechanical regularization. Nucl. Inst. Meth. Phys. Res. B 324, 70-79 (2014)

144. Tasan, C., Hoefnagels, J., Geers, M.: Indentation-based damage quantification revisited. Scripta Mat. 63, 316-319 (2010)

145. Tikhonov, A., Arsenin, V.: Solutions of ill-posed problems. J. Wiley, New York (USA) (1977)

146. Tomičevič, Z., Hild, F., Roux, S.: Mechanics-aided digital image correlation. J. Strain Analysis 48, 330-343 (2013)

147. Voyiadjis, G.Z. (ed.): Handbook of Damage Mechanics. Springer, New York, NY (USA) (2014)

148. Weck, A., Wilkinson, D.S., Maire, E., Toda, H.: Visualization by X-ray tomography of void growth and coalescence leading to fracture in model materials. Acta Mater. 56(12), 2919-2928 (2008)

149. Withers, P., Preuss, M.: Fatigue and damage in structural materials studied by X-ray tomography. Ann. Rev. Mat. Res. 42, 81-103 (2012)

150. Xu, F., Helfen, L., Moffat, A., Johnson, G., Sinclair, I., Baumbach, T.: Synchrotron radiation computed laminography for polymer composite failure studies. J. Synchr. Radiat. 17, 222-226 (2010)

151. Zhang, H., Scholz, A.K., Vion-Loisel, F., Merckel, Y., Brieu, M., Brown, H., Roux, S., Kramer, E., Creton, C.: Opening and closing of nanocavities under cyclic loading in a soft nanocomposite probed by real time small angle X-ray scattering. Macromolecules 46(3), 900-913 (2013) 